\title{
Outflow and Infall in a Sample of Massive Star Forming Regions.
}

\author{
P. D. Klaassen \& C. D. Wilson \\ Dept. of Physics and Astronomy, McMaster University, Hamilton, ON, Canada \\ klaassp@physics.mcmaster.ca
}

\begin{abstract}
We present single pointing observations of $\mathrm{SiO}, \mathrm{HCO}^{+}$and $\mathrm{H}^{13} \mathrm{CO}^{+}$from the James Clerk Maxwell Telescope towards 23 massive star forming regions previously known to contain molecular outflows and ultracompact HII regions. We detected $\mathrm{SiO}$ towards 14 sources and suggest that the non-detections in the other nine sources could be due to those outflows being older and without ongoing shocks to replenish the $\mathrm{SiO}$. We serendipitously detected $\mathrm{SO}_{2}$ towards 17 sources in the same tuning as $\mathrm{HCO}^{+}$. We detected $\mathrm{HCO}^{+}$towards all sources, and suggest that it is tracing infall in nine cases. For seven infall candidates, we estimate mass infall rates between $1 \times 10^{-2}$ and $2 \times 10^{-5} \mathrm{M}_{\odot} \mathrm{yr}^{-1}$. Seven sources show both $\mathrm{SiO}$ detections (young outflows) and $\mathrm{HCO}^{+}$infall signatures. We also find that the abundance of $\mathrm{H}^{13} \mathrm{CO}^{+}$tends to increase along with the abundance of $\mathrm{SiO}$ in sources for which we could determine abundances. We discuss these results with respect to current theories of massive star formation via accretion. From this survey, we suggest that perhaps both models of ionized accretion and halted accretion may be important in describing the evolution of a massive protostar (or protostars) beyond the formation of an HII region.
\end{abstract}

Subject headings: Stars: Formation — ISM: Jets and Outflows - Accretion HII regions — Submillimeter — Molecular Processes

\section{Introduction}

The dynamics in massive star forming regions are, in general, much more complex than in regions which form only low mass stars. For instance, in the early stages within a low mass star forming region, the dynamics can be understood in terms of a few broad categories: large scale infall, which causes a disk to form, accretion through the disk, and outflow to release angular momentum (see for example, Di Francesco et al. 2001, André et al. 1993, Muzerolle et al. 2003). In intermediate and high mass star forming regions, 
turbulence, stellar winds, multiple sites of star formation, and, for regions with massive star formation, the presence of HII regions, all contribute to the dynamics in these regions as well (i.e. Beuther et al. 2006, Shepherd \& Churchwell 1996, McKee \& Tan 2003, Krumholz et al. 2005). The more complicated source dynamics make the processes involved in the formation of the most massive stars much more difficult to understand than those involved in the formation of lower mass stars. Adding to the complexity, massive stars do not form as often as their lower mass counterparts and so we must look to larger distances before finding examples of high mass star formation. For instance, the average distance to the 63 sources in Shirley et al. (2003, hereafter S03) is $5.3 \mathrm{kpc}$.

If we assume massive stars form through accretion, that this accretion occurs in the inner regions of disks (i.e. Pudritz \& Norman 1986, or Shu et al. 1994), and that these disks have radii of a few thousand AU (Chini et al. 2004, Beltran et al. 2004, Cesaroni et al. 2005), we do not yet quite have the resolving power to detect accretion directly at the distances to massive star forming regions (1000 AU at $5.3 \mathrm{kpc}$ is $\sim 0.2^{\prime \prime}$ ). Here, we define accretion as the infall motions from the disk onto the forming star, in contrast to the larger scale motions of envelope material falling onto the disk. However, while we cannot observe accretion directly, its presence can be inferred from the presence of accretion tracers such as larger scale infall and outflow. Infall can act to replenish disk material as mass accretes onto a protostar (Nakamura 2000), while molecular outflows serve as a release mechanism for the angular momentum which builds up during the accretion process (e.g Arce et al. 2006). These large scale motions are seen in star forming regions of all mass scales (see for instance Beuther \& Shepherd 2005).

In massive star forming regions, the accretion rates are orders of magnitude greater than in low mass star forming regions (i.e. Beuther et al. 2002), while the accreted masses are only approximately one order of magnitude greater. These accretion rates and masses result in accretion timescales that are much shorter than in low mass star forming regions, which allows the Kelvin-Helmholtz timescale to become important in the evolution of the protostar (e.g., there is no pre-main sequence stage for massive star formation). The outward radiation and thermal pressure from the forming star becomes strong enough that it can ionize the surrounding medium and a small, highly ionized HII region (either hypercompact (HCHII) or ultracompact (UCHII) region, Keto 2003) can form. It is still unclear whether the outward pressure needed to create the HII region is strong enough to halt accretion, or whether accretion can continue in some form (either through a molecular or ionized disk, or through an ionized accretion flow) after the formation of an HII region. Some models suggest that accretion must halt before the onset of a visible UCHII region (i.e. Garay \& Lizano 1999, Yorke 2002), while other models suggest that an ionized accretion flow can continue through an HII region (i.e. Keto 2003, 2006). 
There is now also observational evidence which suggests that, once the protostar becomes hot enough to ionize its surroundings, both modes of massive star formation (halted and ionized accretion) are possible. G10.6-0.4 has been shown to have an ionized accretion flow by Sollins et al. (2005) and Keto \& Wood (2006), while accretion in G5.89-0.39 seems to have halted at the onset of the UCHII region (Klaassen et al. 2006). Although sample statistics at this point are still quite small, these two examples pose interesting questions. We do not yet have enough data to determine whether the apparently conflicting models of halted and ionized accretion can both be correct. However, we can begin with a uniform survey of infall and outflow tracers in massive star forming regions in order to constrain massive star formation scenarios.

In this paper, we present a survey of 23 massive star forming regions. Because we are interested in the relationship between accretion and outflow after the formation of an HII region, our source selection criteria include (1) the presence of an UCHII region, which indicates that there is a massive protostar forming, and (2) previous evidence of outflows, which suggests ongoing accretion in most formation scenarios. Sources were selected based on inclusion in the Wood \& Churchwell (1989) and Kurtz et al. (1994) catalogs of UCHII regions as well as having molecular outflow signatures in the Plume et al. (1992) survey of massive star forming regions. Additional sources were taken from Hunter (1997) which were shown to have both UCHII regions and molecular outflows.

We describe the observations collected for this survey in Section 2, we discuss the results of these observations in Section 3, and present our conclusions in Section 4 .

\section{Observations}

Observations of $\mathrm{SiO}(\mathrm{J}=8-7), \mathrm{HCO}^{+}$, and $\mathrm{H}^{13} \mathrm{CO}^{+}(\mathrm{J}=4-3)$ were taken at the James Clerk Maxwell Telescope (JCMT) 1 in 2005 (as parts of projects M05AC11 and M05BC04). $\mathrm{SiO}(347.330 \mathrm{GHz})$ and $\mathrm{H}^{13} \mathrm{CO}^{+}(346.999 \mathrm{GHz})$ were observed simultaneously in the same sideband by tuning the receiver to $347.165 \mathrm{GHz}$. Thirteen or twenty minute observations, depending on the source elevation, were taken towards each source with a velocity resolution of $1.08 \mathrm{~km} \mathrm{~s}^{-1}$, which resulted in rms noise levels of $\mathrm{T}_{\mathrm{MB}}<0.07 \mathrm{~K}$. Separately, we observed $\mathrm{HCO}^{+}(356.370 \mathrm{GHz})$ with a velocity resolution of $0.53 \mathrm{~km} \mathrm{~s}^{-1}$ to an rms noise limit of $\mathrm{T}_{\mathrm{MB}}<0.13 \mathrm{~K}$ in twenty minute integrations. Both sets of observations were taken in position

\footnotetext{
${ }^{1}$ The James Clerk Maxwell Telescope is operated by The Joint Astronomy Centre on behalf of the Particle Physics and Astronomy Research Council of the United Kingdom, the Netherlands Organisation for Scientific Research, and the National Research Council of Canada.
} 
switching mode with dual mixers and a sideband rejection filter in place. Table 1 shows the positions, rms noise limits of both tunings, the local standard of rest velocity, and distances to all sources in this survey. The half power beam width for these observations is $15^{\prime \prime}$, and the main beam efficiency is $\eta_{\mathrm{mb}}=0.62$. Data were obtained using the DAS autocorrelator system and reduced using the SPECX software package.

Linear baselines were removed from all spectra except for those towards G10.47. For this source, there were so many different chemical species in the observed spectrum that we were unable to fit a linear baseline over the entire $\sim 700 \mathrm{~km} \mathrm{~s}^{-1}$ bandwidth of the $347 \mathrm{GHz}$ observations or the $\sim 450 \mathrm{~km} \mathrm{~s}^{-1}$ bandwidth of the $356 \mathrm{GHz}$ observations. In this case, no baseline was removed.

We also present $9 \times 9$ maps of one source (G45.07) in the same emission lines. These raster maps are sampled every 5 " and have rms noise limits of 0.10 and $0.14 \mathrm{~K}\left(\mathrm{~T}_{\mathrm{MB}}\right)$ for the 347 and $356 \mathrm{GHz}$ tunings, respectively. Note that these values are different than the ones reported in Table 1 for the single pointing observations. The DAS autocorrelator was configured with the same tunings as were described above for the single pointing observations, and the maps were centered at the same position.

\section{Results}

This survey of single pointing observations towards 23 massive star forming regions is meant as an initial, uniform survey from which to base future observations. With these observations, we can only comment on the molecular gas component within our beams; we cannot discuss the larger scale molecular dynamics, or the ionized gas components of these regions. For our sources, the selection criteria of having an HII region confirms that these regions are forming massive stars.

Figures 1 through 4 show the single pointing $\mathrm{SiO}$ and $\mathrm{HCO}^{+} / \mathrm{H}^{13} \mathrm{CO}^{+}$spectra towards all sources; the spectra are ordered according to $\mathrm{SiO}$ integrated intensity. For each panel in these figures, line brightnesses have been corrected for the JCMT main beam efficiency and centered on the $V_{\mathrm{LSR}}$ of the source (Table 1). Figures 1 and 2 show $\mathrm{SiO}$ and $\mathrm{HCO}^{+} / \mathrm{H}^{13} \mathrm{CO}^{+}$ spectra, respectively, towards the sources with no $\mathrm{SiO}$ detections. Figures 3 and 4 show the $\mathrm{SiO}$ and $\mathrm{HCO}^{+} / \mathrm{H}^{13} \mathrm{CO}^{+}$spectra, respectively, towards sources with $\mathrm{SiO}$ detections. Peak line strengths and integrated intensities are given for all three lines in Table 2.

$\mathrm{SiO}$ was only detected in 14 out of our 23 sources, where we define a detection as a minimum of $4 \sigma$ in integrated intensity. The rms noise limits in integrated intensity were calculated using $\Delta I=T_{\mathrm{rms}} \Delta v \sqrt{N_{\text {chan }}}$ where $T_{\text {rms }}$ is the rms noise level in $\mathrm{K}, \Delta v$ is the velocity 
resolution of the observations, and $N_{\text {chan }}$ is the number of channels over which the integrated intensity is calculated. $\mathrm{HCO}^{+}$was detected in all sources and $\mathrm{H}^{13} \mathrm{CO}^{+}$was detected in all but two sources. Along with $\mathrm{HCO}^{+}$, we serendipitously observed $\mathrm{SO}_{2}\left(\mathrm{~J}=10_{4,6}-10_{3,7}\right.$ at 356.755 $\mathrm{GHz}$ ) in 17 of our sources. The $\mathrm{HCO}^{+}$and $\mathrm{SO}_{2}$ lines are only separated by $17 \mathrm{~km} \mathrm{~s}^{-1}$ and thus the lines were blended in eight sources. For the sources with $\mathrm{SO}_{2}$ detections, we have also plotted (in gray) the lower intensity $\mathrm{HCO}^{+}$observations in order to highlight the $\mathrm{SO}_{2}$ emission (Figures 2 and 4).

Double peaked $\mathrm{HCO}^{+}$line profiles were observed towards 10 sources, with nine of them having stronger blue peaks than red. This blue line asymmetry in an optically thick tracer such as $\mathrm{HCO}^{+}$is often suggestive of infall (i.e Myers et al. 1996). We discuss the possibility of our observations tracing large scale infall further in Section 3.2 .

The distances to our sources, as taken from the literature, are shown in Table 1. The average distance is $5.7 \pm 3.8 \mathrm{kpc}$, where the error quoted reflects the $1 \sigma$ dispersion in the distances. Since our observations were taken with a $15^{\prime \prime}$ beam, this resolution corresponds an average linear size of 0.4 pc for our observations.

$\mathrm{SiO}$ is a well known outflow tracer, since in the general interstellar medium, Si is frozen out onto dust grains. When the gas in a region is shocked (i.e. the gas through which a protostellar outflow is passing) the dust grains can sublimate and $\mathrm{Si}$ is released into the gas phase. After the passage of a shock, the $\mathrm{SiO}$ abundance $\left([\mathrm{SiO}] /\left[\mathrm{H}_{2}\right]\right)$ can jump to almost $10^{-6}$, whereas the dark cloud abundance of $\mathrm{SiO}$ is often closer to $10^{-12}$ (see for example Schilke et al. 1997, Caselli et al. 1997, or van Dishoeck \& Blake, 1998).

While $\mathrm{SiO}$ is easily identified as an outflow tracer, the emitting region for $\mathrm{HCO}^{+}$is much less certain. Many authors suggest that $\mathrm{HCO}^{+}$can be used to trace the envelope material surrounding a protostellar region (i.e. Hogerheijde et al. 1997, Rawlings et al. 2004), while others suggest that it traces disk material (i.e. Dutrey et al. 1997). One thing that is apparent, however, is that it becomes optically thick very quickly and readily self absorbs.

We detected $\mathrm{SO}_{2}$ in 17 of our sources, suggesting that our beam contains at least some molecular gas at temperatures greater than $100 \mathrm{~K}$ (see for instance Doty et al. 2002, Charnley 1997). However, Fontani et al. (2002) have determined the average temperature in twelve massive star forming regions to be $44 \mathrm{~K}$, using observations with beam sizes comparable to those presented here. For seven of our sources, which were observed in the Fontani et al (2002) sample, the average temperature is also $44 \mathrm{~K}$. Thus, in the following analysis, we adopt an ambient temperature of $44 \mathrm{~K}$ for all sources.

Table 3 shows the column densities for each region derived for both $\mathrm{SiO}$ and $\mathrm{H}^{13} \mathrm{CO}^{+}$. The column density was calculated assuming that each tracer is optically thin, in local 
thermodynamic equilibrium, and at an ambient temperature of $44 \mathrm{~K}$. For optically thin lines, the column density of the observed transition scales directly with the integrated intensity of the line (see for instance, Tielens 2005):

$$
N_{u}=\frac{8 k \pi \nu^{2}}{h c^{3}} \frac{1}{A_{u \ell}} \int T_{\mathrm{MB}} d v
$$

where $N_{u}$ is the column density in the upper state of the transition, $A_{u \ell}$ is the Einstein A coefficient, $\nu$ is the frequency of the $\mathrm{J}=u-\ell$ transition, and $\int T_{\mathrm{MB}} d v$ is the integrated intensity of the line. The column density of this one state can then be related to the total column density of that molecule through the partition function. It is the total column density for the molecule (not the observed state) that is presented in Table 3 ,

\subsection{Source Properties derived from SiO observations}

For each source we determined the column density, or upper limit to the column density, in $\mathrm{SiO}$ (Table 3) using the methods described above. These column densities can be compared to the column densities of other molecules (i.e. CS) for the same regions in order to determine the fractional abundance of $\mathrm{SiO}$, if the abundance of the other molecule is known. We were able to obtain the $\mathrm{CS}$ or $\mathrm{C}^{34} \mathrm{~S}$ column densities for fifteen of our sources from the literature. Column densities for fourteen sources were taken from Plume et al. (1997, hereafter P97), with the column density for one additional source taken from Wang et al. (1993). For those sources with $\mathrm{C}^{34} \mathrm{~S}$ column densities instead of CS column densities, we assumed an abundance ratio of $[\mathrm{CS}] /\left[\mathrm{C}^{34} \mathrm{~S}\right]=22$ (Wilson \& Rood, 1994) to determine a CS column density. The abundance of CS, relative to $\mathrm{H}_{2}$, was calculated by $\mathrm{S} 03$ for 13 of these sources, and we assume a CS abundance of $1.2 \times 10^{-9}$ for the other two source for which the CS column density is known, since this was the average CS abundance as calculated by S03. We then compare the column density and abundance of CS to our observed $\mathrm{SiO}$ column density, or column density upper limit, to determine the abundance of $\mathrm{SiO}$ relative to $\mathrm{H}_{2}$ in our sources (Table 3).

Despite our source selection criteria requiring previous evidence of outflows, we detected $\mathrm{SiO}$ towards only 14 of our 23 sources. This raises a number of questions, such as: is the observed $\mathrm{SiO}$ in fact tracing outflow if we do not detect it in all sources? Why do we not detect $\mathrm{SiO}$ in all sources? Is the signal being beam diluted at large distances? Has the $\mathrm{Si}$ evolved into other species? Below we first address whether the detected $\mathrm{SiO}$ can be used

as an outflow tracer, and then discuss reasons for our non-detections of $\mathrm{SiO}$ in nine of our sources. 
Si is liberated in shocks, and if these shocks are not due to the outflow, they must be due to the photo dissociation region (PDR) surrounding the UCHII region. Evidence for diffuse (not collimated) $\mathrm{SiO}$ can be seen in W75N (Shepherd, Kurtz \& Testi, 2004) suggesting that the $\mathrm{SiO}$ may be due to the PDR and not an outflow. Models and observations of $\mathrm{SiO}$ in the PDRs around high mass star forming regions suggest moderate $\mathrm{SiO}$ enhancement, and that the $\mathrm{SiO}$ abundance is independent of the ambient radiation field (i.e. Schilke et al. 2001). Schilke et al. (2001) find $\mathrm{SiO}$ column densities of $\sim 10^{12} \mathrm{~cm}^{-2}$ in their observed PDRs. This is, admittedly, below our detection threshold; however, we detect average $\mathrm{SiO}$ column densities of $\sim 10^{14} \mathrm{~cm}^{-2}$. This suggests a possibly higher SiO abundance than found in PDRs.

The enhanced $\mathrm{SiO}$ column density alone is not enough to discount the origin of the $\mathrm{SiO}$ in our sources as the PDR and so we can consider how our $\mathrm{SiO}$ abundance varies with the ambient radiation field. For the gas near an HII region, we can approximate the strength of the ambient radiation field using the Far Infrared (FIR) luminosity of the region. For twelve of our sources with $\mathrm{SiO}$ detections and abundance calculations, we obtained the FIR luminosity from either Wood \& Churchwell (1989), Kurtz et al. (1994) or Evans et al. (1981). We then scaled their values for the different source distances used in this study (see Table 3). Comparing the $\mathrm{SiO}$ abundance to the source luminosity (see Figure 5), we find that the $\mathrm{SiO}$ abundance increases with source luminosity. There is a $10 \%$ chance that this relationship could arise from uncorrelated data. Thus, it is possible that our result is contrary to the findings of Schilke et al (2001), and we suggest that the $\mathrm{SiO}$ we observe does come primarily from outflow shocks.

We can also compare our detection rate of $\mathrm{SiO}$ to that of the $\mathrm{SiO}$ survey towards maser sources of Harju et al (1998) who observed $\mathrm{SiO}(\mathrm{J}=2-1)$ and $\mathrm{SiO}(\mathrm{J}=3-2)$. For comparison to our results, we only consider the sources in Harju et al. which are listed as UCHII regions. Our detection rate is $61 \%$, compared to their rate of $29 \%$. While our detection threshold is slightly lower (our observations have rms noise levels generally below $0.05 \mathrm{~K}$ at $347 \mathrm{GHz}$, while their rms noise levels are generally below $0.08 \mathrm{~K}$ ), we suggest that the different detection rates are due to differences in the source selection criteria. Although both samples contain UCHII regions, our sample contains sources with previous observations of outflows, while Harju et al. have selected sources based on previous observations of masers. For the 12 sources which overlap between the two studies, we detect $\mathrm{SiO}$ towards 9 sources, while they detect $\mathrm{SiO}$ towards 10 . They detected $\mathrm{SiO}(\mathrm{J}=2-1)$ in G31.41 while we did not detect it in $\mathrm{SiO}(\mathrm{J}=8-7)$.

Based on this comparison to $\mathrm{SiO}$ observations of UCHII regions not selected by outflows, which have a lower $\mathrm{SiO}$ detection rate, and that our $\mathrm{SiO}$ abundance increases with source 
luminosity, we suggest that the $\mathrm{SiO}$, in the 14 sources in which it is detected, is being generated in the outflow. Previous, high resolution observations of $\mathrm{SiO}$ also suggest that $\mathrm{SiO}$ can be enhanced in the outflows from high mass stars (i.e. Beuther et al. 2004, Beuther, Schilke \& Gueth 2004) just as it is in the outflows from low mass stars.

The nine non-detections in our sample could be caused by beam dilution if these sources are on average further away. However, if we compare the distances for sources with and without $\mathrm{SiO}$ detections, we find average distances of $6.3 \pm 4.4$ and $4.4 \pm 2.5 \mathrm{kpc}$, respectively. Thus, the non-detections cannot be attributed to larger average source distances and so beam dilution can play only a minimal role in the non-detections of $\mathrm{SiO}$.

If these $\mathrm{SiO}$ non-detections are not due to distance effects, there must be some local phenomenon which can explain why $\mathrm{SiO}$ is not being detected in regions known to contain protostellar outflows. It is possible that the $\mathrm{Si}$ is evolving into different species and the $\mathrm{SiO}$ abundance is dropping back down to dark cloud values. Pineau des Forêts et al. (1997) suggest that a few $\times 10^{4}$ yr after the $\mathrm{Si}$ is liberated from dust grains and forms $\mathrm{SiO}$, it can either freeze out back onto dust grains or oxidize and form $\mathrm{SiO}_{2}$. Thus, the lack of $\mathrm{SiO}$ may be due to silicon moving into other species if it was liberated more than $10^{4} \mathrm{yr}$ ago. This interpretation implies that we did not detect $\mathrm{SiO}$ in some of our sources because the outflow generating mechanism shut off more than $10^{4}$ years ago, and the outflow observed in $\mathrm{HCO}^{+}$ (or in other molecules by other authors) is a remnant of previous accretion.

The kinematic ages of nine of our sources are listed in the Wu et al (2004) catalog of high velocity outflows. Of these nine sources, five were also included in the P97 and S03 studies. This results in five sources for which we have both the kinematic age of the outflow, and the abundance of $\mathrm{SiO}$. The relationship between outflow age and $\mathrm{SiO}$ abundance is shown in Figure 6 along with the model predictions of Pineau des Forêts et al. (1997). With only five points, it is difficult to draw conclusions about the relationship between outflow age and $\mathrm{SiO}$ abundance, especially given the uncertain beam filling factor. At higher resolution, these points would likely move upwards to higher abundances. A larger beam filling factor would move the points for the two young outflows (G5.89 and Cep A) towards the model predictions. As for the other three outflows, this would move them further from the model predictions. We suggest that the outflow generating mechanism is continuing to shock these regions, replenishing the $\mathrm{SiO}$. The oldest of these sources (G192.58) shows an infall signature in $\mathrm{HCO}^{+}$, which also suggests that the outflow is still being powered. 


\subsection{Source properties derived from $\mathrm{HCO}^{+}$observations}

Given the large average distance to our sources $(5.7 \mathrm{kpc})$, our $15^{\prime \prime}$ beam subtends an average linear distance of $0.4 \mathrm{pc}$. Thus, it is quite likely that the $\mathrm{HCO}^{+}$emitting region does not entirely fill our single JCMT beam. In general we can determine the beam filling factor, $f$, for each source using

$$
T_{L}=f_{\text {source }} T_{s}\left(1-e^{-\tau}\right)
$$

where $T_{L}$ is the line brightness temperature measured at the telescope (corrected for telescope efficiencies) and $T_{s}$ is an approximation to the ambient temperature $(44 \mathrm{~K})$ which is valid at densities greater than $\sim 10^{3} \mathrm{~cm}^{-3}$ (Rholfs \& Wilson, 1994). In a number of our sources, the $\mathrm{HCO}^{+}$is asymmetric, and thus cannot be consistently used to determine a beam filling factor. Instead, we can use the optically thin $\mathrm{H}^{13} \mathrm{CO}^{+}$line, and we can simplify the above equation to $f=T_{L} /\left(T_{s} \tau\right)$. These values are shown in Table 3 ,

$\mathrm{HCO}^{+}$becomes optically thick quite quickly due to its relatively high abundance with respect to $\mathrm{H}_{2}\left(\left[\mathrm{HCO}^{+}\right] /\left[\mathrm{H}_{2}\right] \sim 10^{-8}\right)$, and as such, can be used to roughly trace outflow and to trace infall (i.e. Myers et al. 1996) if the line profile shows a double peak. We determined the optical depth of $\mathrm{HCO}^{+}$towards each source using Equation 1 of Choi et al. (1993), and found that in all but one case (G139.9), it is optically thick. In all cases, the optical depth of $\mathrm{HCO}^{+}$is less than 77 (the abundance ratio between $\mathrm{HCO}^{+}$and $\mathrm{H}^{13} \mathrm{CO}^{+}$, Wilson \& Rood, 1994), resulting in optically thin $\mathrm{H}^{13} \mathrm{CO}^{+}$towards all sources. Because $\mathrm{HCO}^{+}$is optically thick in its line center, the line wings can be used to detect outflows, and so, if there is an outflow, it should be detectable in $\mathrm{HCO}^{+}$even if it goes undetected in $\mathrm{SiO}$. Gaussian profiles were fit to our $\mathrm{HCO}^{+}$spectra (either single or double Gaussians, depending on the observed line shape), and the fits were subsequently subtracted from the spectra to leave only the residual outflowing gas. When using two Gaussians to fit the self absorbed spectra, we employed a method similar to the single Gaussian fitting of Purcell et al. (2006) because we used the sides of the detected lines to fit our profiles (see their Figure 3). Comparing the two Gaussian fits to single Gaussian fits showed no significant differences in distinguishing line wing intensity. Because of the possibility of contamination from $\mathrm{SO}_{2}$ emission in the blue shifted outflow wing (at $-17 \mathrm{~km} \mathrm{~s}^{-1}$ ), the peak brightness of the residual emission was determined using only the red shifted wing emission. In all cases (except for G10.47 for which we could not find a linear baseline), we found a minimum of a $5 \sigma$ peak brightness temperature in the residual line wing emission, with 19 of our sources having a minimum of a $10 \sigma$ peak. This result suggests that we can detect outflow motions in all sources using our detections of $\mathrm{HCO}^{+}$, despite not detecting $\mathrm{SiO}$ towards every source. 
Our observations show that for ten of our sources, the spectral line profile of the $\mathrm{HCO}^{+}$ emission has a double peak. This profile could either be due to self absorption of the optically thick $\mathrm{HCO}^{+}$line or from multiple velocity components within our $15^{\prime \prime}$ beam. To break this degeneracy, we observed the optically thin $\mathrm{H}^{13} \mathrm{CO}^{+}$. If the $\mathrm{H}^{13} \mathrm{CO}^{+}$line has a single peak at the same velocity as the $\mathrm{HCO}^{+}$absorption feature, then it is likely that the $\mathrm{HCO}^{+}$line is self absorbed. If, however, the $\mathrm{H}^{13} \mathrm{CO}^{+}$also has two peaks, and they are at approximately the same velocities as the two $\mathrm{HCO}^{+}$peaks, it would suggest that there are multiple components within the beam. Of our ten sources with double $\mathrm{HCO}^{+}$peaks, only one shows a double peak profile in $\mathrm{H}^{13} \mathrm{CO}^{+}$(G20.08). This results in nine sources with double peaked optically thick $\mathrm{HCO}^{+}$.

In addition to the nine optically thick sources, similar line asymmetries appear in a number of other sources. However, in these sources, there is no clear emission gap producing a double peak profile, only an emission shoulder (i.e. De Vries \& Myers 2005). If we take G75.78 as an example, the $\mathrm{HCO}^{+}$line peak is red shifted from the rest velocity of the source, with a blue shifted emission shoulder.

There are a number of different kinds of source dynamics that can lead to the double peaked line profiles seen in our spectra, such as infall, outflow and even rotation. However, infall is the only one of these processes which would produce line asymmetries which are consistently blue (i.e. the blue peak is higher than the red peak or shoulder). If these profiles were due to outflow or rotation, there would be no statistical reason to have more sources with higher blue peaks than red peaks. Many previous studies have investigated the statistical significance of using this type of optically thick blue line asymmetry to trace infall as opposed to other dynamical motions (i.e. Mardones et al. 1997 and Gregersen et al. 1997 for low mass star forming regions, and Fuller et al. 2005 for high mass star forming regions).

Of the 10 sources in our survey which have double peaked $\mathrm{HCO}^{+}$profiles, we suggest eight may be indicative of infall. The other two sources are G20.08 and G45.47. G20.08 has already been shown to have multiple components in the beam from the double peaked $\mathrm{H}^{13} \mathrm{CO}^{+}$profile, and $\mathrm{G} 45.47$ has a brighter red peak than blue. There are two additional sources (G19.61, and G240.3) in which $\mathrm{HCO}^{+}$has a strong red shifted shoulder, which we suggest may also be tracing infall. This analysis gives a total of ten infall candidates in our sample of 23 sources.

A recent survey of $\mathrm{HCO}^{+}(\mathrm{J}=1-0)$ towards sources with methanol masers shows an even distribution of sources with blue and red line asymmetries, and a higher percentage of self absorbed lines than in our study (Purcell et al. 2006). Of the six sources which overlap between our survey and that of Purcell et al, all six are self absorbed in $\mathrm{HCO}^{+}(\mathrm{J}=1-0)$. Five of them have blue line asymmetries consistent with infall, while only one (G31.41) has 
its red peak brighter than its blue peak. We only find self-absorption in $\mathrm{HCO}^{+}(\mathrm{J}=4-3)$ for three of these six sources. In two of the sources for which we do not see a clear self-absorption feature, we do see evidence for a red shifted shoulder which may be showing unresolved infall.

The sources in Purcell et al. (2006) have an even distribution of red and blue line asymmetries, while we have a clear bias towards detecting blue line asymmetries. This comparison could suggest that the higher energy $\mathrm{J}=4-3$ transition of $\mathrm{HCO}^{+}$is a better tracer of infalling gas because it does not self absorb as readily as the $\mathrm{J}=1-0$ transition.

For each of the 8 sources with blue, double peaked $\mathrm{HCO}^{+}$profiles, we can determine an infall velocity $\left(v_{\text {in }}\right)$ using the two layer radiative transfer model of Myers et al. (1996). Using their equation 9, we find infall velocities for all eight double peaked infall sources (Table 4). The mass infall rate can then be determined using:

$$
\dot{M}=\frac{d M}{d t} \approx \frac{M}{t}=\frac{\rho V v_{\mathrm{in}}}{r_{\mathrm{gm}}}=\frac{4}{3} \pi n_{\mathrm{H}_{2}} \mu m_{\mathrm{H}} r_{\mathrm{gm}}^{2} v_{\mathrm{in}}
$$

where $\mu$ is the mean molecular weight $(\mu=2.35)$, the geometric mean radius $\left(r_{\mathrm{gm}}\right)$ is the unresolved circular radius of the $\mathrm{HCO}^{+}$emitting region derived from the beam radius and the beam filling factor $\left(r_{\mathrm{gm}}=\sqrt{f} r_{\text {beam }}\right)$, and $n_{\mathrm{H}_{2}}$ is the ambient source density. For seven of the sources in Table 4, the ambient density was determined by either P97, Hofner et al. (2000), or Wang et al. (1993). We could not find the ambient density for the eighth source (G192.58). From this analysis, we determine mass infall rates ranging from $1 \times 10^{-2}$ to $2 \times 10^{-5} \mathrm{M}_{\odot} \mathrm{yr}^{-1}$. These values are slightly higher than those generally observed for low mass star forming regions, but are consistent with the accretion rates derived for high mass star forming regions by McKee \& Tan (2003). Since outflow rates are orders of magnitude higher in high mass star forming regions (i.e. Beuther et al. 2002) it is not unreasonable to suggest that infall rates are also much higher in these regions.

The mass outflow rate for only one of these sources (Cep A) can be determined from the $\mathrm{Wu}$ et al. (2004) survey of high velocity outflows by dividing the mass in the outflow by the kinematic age of the outflow. We find the ratio of the mass outflow rate to the mass infall rate to be $\dot{M}_{\text {out }} / \dot{M}_{\text {in }} \approx 16$. This value is only slightly higher than values seen in other high mass star forming regions (i.e. Behrend \& Maeder 2001). Also, models suggest that a mass equivalent to $20-30 \%$ of the mass accreted onto a protostar is ejected as a wind (i.e. Pelletier \& Pudritz 1992, Shu et al. 1994), and that this wind entrains 5-20 times its mass in the outflow (Matzner \& McKee 1999). 


\subsection{Source properties derived from mapping G45.07}

At a distance of $9.7 \mathrm{kpc}$, G45.07 is one of our furthest sources. This source was known from previous observations to have multiple continuum sources (De Buizer et al. 2003, 2005). The three continuum sources were observed in the mid-Infrared (MIR) and all three fall within $6^{\prime \prime}$ of our map center. A fairly young outflow has also been mapped at high resolution ( $<3^{\prime \prime}$ synthesized beam) in CO and CS towards this region (Hunter et al. 1997). They observed a bipolar outflow with a position angle of $-30^{\circ}$ (east of north), as well as a red shifted absorption feature in their CS observations which they take to be indicative of infall.

Due to the large distance to this source, we should be able to detect all of the emission associated with this source in a fairly small map. In the left panel of Figure 7 we present a map of the $\mathrm{SiO}$ (contours) and $\mathrm{H}^{13} \mathrm{CO}^{+}$(halftone) emission in this region. The right panel of Figure 7 shows the $\mathrm{HCO}^{+}$emission. In both figures, the $5 \sigma\left(2.1 \mathrm{~K} \mathrm{~km} \mathrm{~s}^{-1}\right) \mathrm{H}^{13} \mathrm{CO}^{+}$ emission contour is plotted as a dashed line to help guide the eye. The first contour for $\mathrm{SiO}$ in the left panel and only contour of $\mathrm{HCO}^{+}$in the right panel are also $5 \sigma(2.2$ and $3.5 \mathrm{~K}$ $\mathrm{km} \mathrm{s}^{-1}$ respectively). The differences in the $5 \sigma$ contour levels for each tracer come from the different single channel rms levels between the two tunings, and the width of each line as given in Table 2. Also plotted in both figures are the three MIR continuum sources observed by De Buizer et al (2003, 2005).

If we did not have the added information provided by this map, there would be two main conclusions we could draw from our single pointing observations towards this source. The first is that the enhanced blue emission in all three tracers suggests that our pointing is observing more of the blue shifted outflow lobe than the red. Second, since we only have one peak in the spectrum of each tracer, there is only one source and we cannot classify it as infalling.

The left panel of Figure[7] shows contours of $\mathrm{SiO}$ emission superimposed on the $\mathrm{H}^{13} \mathrm{CO}^{+}$ halftone. With beam spacings of $5^{\prime \prime}$, these maps are oversampled; however we note that much of the structure in the $\mathrm{SiO}$ emission is on scales comparable to the size of the JCMT beam. For instance, the structure at $\Delta \alpha=-10^{\prime \prime}, \Delta \delta=0^{\prime \prime}$ is offset from the map center by more than the radius of our beam and could be independent from the emission at the map center. There is also $\mathrm{SiO}$ emission at $\Delta \alpha=5^{\prime \prime}, \Delta \delta=15^{\prime \prime}$, which is more than a full beam away from the map center, and suggests that the $\mathrm{SiO}$ emission is more extended that the primary beam of our observations. In fact, it appears as though there is a second $\mathrm{SiO}$ emission peak towards the upper left of the left panel of Figure 7. Interestingly, there does not appear to be as much $\mathrm{H}^{13} \mathrm{CO}^{+}$emission at this northern position. This comparison shows that the $\mathrm{SiO}$ and $\mathrm{H}^{13} \mathrm{CO}^{+}$lines are tracing different gas populations in this region. The excess $\mathrm{SiO}$ 
emission is offset from the map center in the same direction as the CO emission shown in Hunter et al. (1997) at much higher resolution.

The right panel of Figure 7 shows the $\mathrm{HCO}^{+}$emission for this region. It appears that the $\mathrm{HCO}^{+}$emission extends much further than the $\mathrm{SiO}$ emission, suggesting it is tracing the larger scale envelope material. The line through the middle of this plot indicates the cut taken for the position-velocity (PV) diagram along the outflow axis as described by Hunter et al. (1997)

The two panels of Figure 8 show the $\mathrm{PV}$ diagrams for $\mathrm{SiO}$ and $\mathrm{HCO}^{+}$in our maps both perpendicular and parallel to the outflow axis defined by Hunter et al. (1997). Our single pointing $\mathrm{HCO}^{+}$spectrum (Figure 4) suggests we are observing more blue shifted outflow emission than red shifted emission; however, from our PV diagrams, we see that there is excess blue emission at all positions in our map. This excess blue emission cannot be due to outflow alone; instead, it could be due to an inherent velocity shift between the three continuum sources in our beam. We can, in fact, fit three Gaussian components to most of our $\mathrm{HCO}^{+}$spectra. These Gaussians peak at velocities of 60,52 and $44 \mathrm{~km} \mathrm{~s}^{-1}$, with the peak temperature for each component decreasing with velocity. The third component (at 44 $\mathrm{km} \mathrm{s}^{-1}$ ) could not be fit at all positions because it was intrinsically weaker than the other two peaks, and was lost in the noise towards the edges of the map. It appears as though this third component might be contamination from $\mathrm{SO}_{2}$, which should occur at an apparent velocity of $41 \mathrm{~km} \mathrm{~s}^{-1}$ (or $-17 \mathrm{~km} \mathrm{~s}^{-1}$ in Figure 4).

Perpendicular to the outflow axis, the mean velocity of the $\mathrm{HCO}^{+}$line peak appears to shift from $\sim 58 \mathrm{~km} \mathrm{~s}^{-1}$ at an offset of $+15^{\prime \prime}$ from the source center to $\sim 61 \mathrm{~km} \mathrm{~s}^{-1}$ at an offset of $-15^{\prime \prime}$ from the source (Figure 8). Given our velocity resolution (1.08 $\mathrm{km} \mathrm{s}^{-1}$ ) and spatial resolution $\left(15^{\prime \prime}\right)$, is unclear whether this velocity shift is real. If it is, it could indicate large scale $(\sim 1.4 \mathrm{pc})$ rotation within the core, on a much larger scale than would be expected for a rotating accretion disk.

\subsection{Correlations between Datasets}

Previously, we discussed the reasons why we do not detect $\mathrm{SiO}$ towards a number of our sources, and have calculated the mass infall rates for the sources with double peaks in their $\mathrm{HCO}^{+}$emission, but we have not yet discussed the correlations between the two species. In Figure 9 we plot the logarithm of the abundance of $\mathrm{H}^{13} \mathrm{CO}^{+}$against the same quantity for $\mathrm{SiO}$ (open circles), as well as the column densities of both species (filled circles). The abundance of $\mathrm{H}^{13} \mathrm{CO}^{+}$was calculated in the same manner as the abundance of $\mathrm{SiO}$ 
described above (using the CS column density from P97 and the CS abundance from S03). The probability of obtaining these correlations if the data are, in fact, uncorrelated is $6 \times 10^{-3}$ for the abundances, and $2 \times 10^{-4}$ for the column densities.

As stated earlier, $\mathrm{SiO}$ is a well known shock tracer, and as such, an increased abundance of $\mathrm{SiO}$ would suggest more shocked material within our $15^{\prime \prime}$ beam. The (generally) infall tracing $\mathrm{HCO}^{+}$has been shown by some authors to be destroyed in strong shocks (i.e. Bergin et al. 1998, Jørgensen et al. 2004). However, Wolfire \& Königl (1993) suggest that HCO ${ }^{+}$ can be enhanced in regions with high energy shocks, where electron abundances are much greater. This enhancement in the electron abundance increases the formation rate of ions, and we suggest that this is responsible for the $\mathrm{H}^{13} \mathrm{CO}^{+}$abundance enhancement in our sources. This correlation between the abundances of $\mathrm{H}^{13} \mathrm{CO}^{+}$and $\mathrm{SiO}$ suggests that $\mathrm{HCO}^{+}$ and $\mathrm{H}^{13} \mathrm{CO}^{+}$are not only tracing infalling gas, but also the outflowing gas as well. This conclusion is supported by the strong, and broad, line wing emission detected in $\mathrm{HCO}^{+}$(See Section 3.2 .

$\mathrm{HCO}^{+}$over abundances have been seen in high mass star forming regions not included in this study like NGC 2071 (Girart et al. 1999) and Orion IRc2 (Vogel et al. 1984). In these two papers, the over abundances of $\mathrm{H}^{13} \mathrm{CO}^{+}$are with respect to ambient cloud tracers

such as $\mathrm{CO}$ and $\mathrm{H}_{2}$, rather than the high density or shock tracers like the CS and $\mathrm{SiO}$ with which we are comparing our $\mathrm{H}^{13} \mathrm{CO}^{+}$abundances. However, Viti \& Williams (1999a,b) show that $\mathrm{HCO}^{+}$is indeed over abundant with respect to $\mathrm{CS}$ in the gas surrounding $\mathrm{HH}$ objects, and Jiménez-Serra et al. (2006) also show that the abundance of $\mathrm{H}^{13} \mathrm{CO}^{+}$can be enhanced with respect to $\mathrm{SiO}$ by up to a factor of ten in the same regions ahead of $\mathrm{HH}$ objects.

\section{Discussion and Conclusions}

Without maps of each region, it is impossible to tell how much of the $\mathrm{HCO}^{+}$emission in the line center and in the line wings is due directly to infall and outflow motions; however, based on the arguments we have presented above, we suggest that ten sources show infall motions, and all 23 source show outflow motions based on the $\mathrm{HCO}^{+}$line profiles. We have found evidence for recent outflow activity ( $\mathrm{SiO}$ emission) in 14 out of our 23 sources. Seven of these outflow and infall sources overlap. M17S, G192.6 and G240 appear to show only infall signatures and no $\mathrm{SiO}$ outflow signatures. They do, however, appear to have $\mathrm{HCO}^{+}$ outflow signatures of a minimum of $8 \sigma$.

Detection of line wing emission in $\mathrm{HCO}^{+}$and the relationship between $\mathrm{H}^{13} \mathrm{CO}^{+}$and $\mathrm{SiO}$ abundances described in the previous section suggest that while $\mathrm{SiO}$ is tracing outflow 
in most sources and $\mathrm{HCO}^{+}$is tracing infall in some sources, $\mathrm{HCO}^{+}$is also observable in the outflowing gas for all regions.

We find that the non-detection of $\mathrm{SiO}$ in nine of our sources is not due to beam dilution or larger average distances to the source, but possibly to older outflows for which the Si has likely either frozen back onto dust grains or evolved into $\mathrm{SiO}_{2}$. For these sources, it appears that the accretion may have ceased, and the observed outflowing gas is a remnant of previous accretion.

We have found seven sources with $\mathrm{SiO}$ outflow signatures but no infall signatures in $\mathrm{HCO}^{+}$. This result could be due to a number of factors such as beam dilution of the infalling gas which masks the spectral line profile we would expect for large scale infall.

It is possible that, as the outflow ages and widens, it may impinge on the region in which we could detect infalling gas. For outflow cones oriented along the line of sight, the younger, narrower outflows would have infalling gas with large line of sight velocities and be likely to produce an observable infall signature. However, for the older outflows which have widened, the largest infall velocities will be in the plane of the sky, and unobservable at the resolution of the JCMT. However, this effect would not be as pronounced for outflows in the plane of the sky (such as G5.89, for which we do not see an infall signature). It is difficult to assess the importance of this effect without detailed information on the outflow orientation in each source.

Thus, we suggest that some of these sources may have finished accreting, and what we observe are remnant outflows from a previous phase of accretion. This scenario was suggested by Klaassen et al. (2006) to explain the large scale outflow in G5.89, and this source is one of these seven sources with an $\mathrm{SiO}$ outflow and no apparent infall signature.

The seven sources which show recent outflow activity (those with $\mathrm{SiO}$ emission) and which appear to be undergoing infall are suggestive of ongoing accretion beyond the onset of the HII region. If accretion is ongoing in the presence of an HII region, then it seems likely that this accretion flow may be ionized. This ionized accretion scenario could be similar to low angular momentum accretion with high ionization as suggested by Keto (2006). Thus, from this survey, we suggest that both models of ionized accretion and halted accretion may be important in describing the evolution of a massive protostar (or protostars) beyond the formation of an HII region.

We would like to acknowledge the support of the National Science and Engineering Research Council of Canada (NSERC). We thanks the referee for helpful comments which improved the paper. P.D.K would also like to thank E. Keto and D. Johnstone for helpful 
discussions during the preparation of this manuscript.

\section{REFERENCES}

Andre, P., Ward-Thompson, D., \& Barsony, M. 1993, ApJ, 406, 122

Arce, H. G., Shepherd, D., Gueth, F., Lee, C.-F., Bachiller, R., Rosen, A., \& Beuther, H. 2006, ArXiv Astrophysics e-prints, arXiv:astro-ph/0603071

Behrend, R., \& Maeder, A. 2001, A\&A, 373, 190

Beltrán, M. T., Cesaroni, R., Neri, R., Codella, C., Furuya, R. S., Testi, L., \& Olmi, L. 2004, ApJ, 601, L187

Bergin, E. A., Neufeld, D. A., \& Melnick, G. J. 1998, ApJ, 499, 777

Beuther, H., Schilke, P., Sridharan, T. K., Menten, K. M., Walmsley, C. M., \& Wyrowski, F. 2002, A\&A, 383, 892

Beuther, H., et al. 2004, ApJ, 616, L23

Beuther, H., Schilke, P., \& Gueth, F. 2004, ApJ, 608, 330

Beuther, H., Churchwell, E. B., McKee, C. F., \& Tan, J. C. 2006, ArXiv Astrophysics e-prints, arXiv:astro-ph/0602012

Beuther, H., \& Shepherd, D. 2005, Cores to Clusters: Star Formation with Next Generation Telescopes, 105

Caselli, P., Hartquist, T. W., \& Havnes, O. 1997, A\&A, 322, 296

Cesaroni, R., Neri, R., Olmi, L., Testi, L., Walmsley, C. M., \& Hofner, P. 2005, A\&A, 434, 1039

Charnley, S. B. 1997, ApJ, 481, 396

Chini, R., Hoffmeister, V., Kimeswenger, S., Nielbock, M., Nürnberger, D., Schmidtobreick, L., \& Sterzik, M. 2004, Nature, 429, 155

Choi, M., Evans, N. J., II, \& Jaffe, D. T. 1993, ApJ, 417, 624

Churchwell, E., Walmsley, C. M., \& Cesaroni, R. 1990, A\&AS, 83, 119

De Buizer, J. M., Osorio, M., \& Calvet, N. 2005, ApJ, 635, 452 
De Buizer, J. M., Radomski, J. T., Telesco, C. M., \& Piña, R. K. 2003, ApJ, 598, 1127

De Vries, C. H., \& Myers, P. C. 2005, ApJ, 620, 800

Di Francesco, J., Myers, P. C., Wilner, D. J., Ohashi, N., \& Mardones, D. 2001, ApJ, 562, 770

Doty, S. D., van Dishoeck, E. F., van der Tak, F. F. S., \& Boonman, A. M. S. 2002, A\&A, 389, 446

Dutrey, A., Guilloteau, S., \& Guelin, M. 1997, A\&A, 317, L55

Evans, N. J., II, et al. 1981, ApJ, 244, 115

Fontani, F., Cesaroni, R., Caselli, P., \& Olmi, L. 2002, A\&A, 389, 603

Fuller, G. A., Williams, S. J., \& Sridharan, T. K. 2005, A\&A, 442, 949

Garay, G., \& Lizano, S. 1999, PASP, 111, 1049

Girart, J. M., Ho, P. T. P., Rudolph, A. L., Estalella, R., Wilner, D. J., \& Chernin, L. M. 1999, ApJ, 522, 921

Gregersen, E. M., Evans, N. J., II, Zhou, S., \& Choi, M. 1997, ApJ, 484, 256

Hanson, M. M., Luhman, K. L., \& Rieke, G. H. 2002, ApJS, 138, 35

Harju, J., Lehtinen, K., Booth, R. S., \& Zinchenko, I. 1998, A\&AS, 132, 211

Hofner, P., Wyrowski, F., Walmsley, C. M., \& Churchwell, E. 2000, ApJ, 536, 393

Hogerheijde, M. R., van Dishoeck, E. F., Blake, G. A., \& van Langevelde, H. J. 1997, ApJ, 489, 293

Hunter, T. R. 1997, Ph.D. Thesis,

Hunter, T. R., Phillips, T. G., \& Menten, K. M. 1997, ApJ, 478, 283

Jiménez-Serra, I., Martín-Pintado, J., Viti, S., Martín, S., Rodríguez-Franco, A., Faure, A., \& Tennyson, J. 2006, ApJ, 650, L135

Jørgensen, J. K., Schöier, F. L., \& van Dishoeck, E. F. 2004, A\&A, 416, 603

Keto, E., \& Wood, K. 2006, ApJ, 637, 850

Keto, E. 2003, ApJ, 599, 1196 
Keto, E. 2006, ArXiv Astrophysics e-prints, arXiv:astro-ph/0603856

Klaassen, P. D., Plume, R., Ouyed, R., von Benda-Beckmann, A. M., \& Di Francesco, J. 2006, ApJ, 648, 1079

Krumholz, M. R., McKee, C. F., \& Klein, R. I. 2005, Nature, 438, 332

Kumar, M. S. N., Fernandes, A. J. L., Hunter, T. R., Davis, C. J., \& Kurtz, S. 2003, A\&A, 412,175

Kurtz, S., Churchwell, E., \& Wood, D. O. S. 1994, ApJS, 91, 659

Mardones, D., Myers, P. C., Tafalla, M., Wilner, D. J., Bachiller, R., \& Garay, G. 1997, ApJ, 489, 719

Matzner, C. D., \& McKee, C. F. 1999, Star Formation 1999, Proceedings of Star Formation 1999, held in Nagoya, Japan, June 21 - 25, 1999, Editor: T. Nakamoto, Nobeyama Radio Observatory, p. 353-357, 353

McKee, C. F., \& Tan, J. C. 2003, ApJ, 585, 850

Muzerolle, J., Calvet, N., Hartmann, L., \& D’Alessio, P. 2003, ApJ, 597, L149

Myers, P. C., Mardones, D., Tafalla, M., Williams, J. P., \& Wilner, D. J. 1996, ApJ, 465, L133

Nakamura, F. 2000, ApJ, 543, 291

Pelletier, G., \& Pudritz, R. E. 1992, ApJ, 394, 117

Pineau des Forets, G., Flower, D. R., \& Chieze, J.-P. 1997, IAU Symp. 182: Herbig-Haro Flows and the Birth of Stars, 182, 199

Plume, R., Jaffe, D. T., Evans, N. J., II, Martin-Pintado, J., \& Gomez-Gonzalez, J. 1997, ApJ, 476, 730

Plume, R., Jaffe, D. T., \& Evans, N. J., II 1992, ApJS, 78, 505

Pudritz, R. E., \& Norman, C. A. 1986, ApJ, 301, 571

Purcell, C. R., et al. 2006, MNRAS, 367, 553

Rawlings, J. M. C., Redman, M. P., Keto, E., \& Williams, D. A. 2004, MNRAS, 351, 1054

Rholfs, K., Wilson, T. L. 2004, Tools of Radio Astronomy (4th ed; Berlin; Springer) 
Schilke, P., Walmsley, C. M., Pineau des Forets, G., \& Flower, D. R. 1997, A\&A, 321, 293

Schilke, P., Pineau des Forêts, G., Walmsley, C. M., \& Martín-Pintado, J. 2001, A\&A, 372, 291

Shepherd, D. S., \& Churchwell, E. 1996, ApJ, 472, 225

Shepherd, D. S., Kurtz, S. E., \& Testi, L. 2004, ApJ, 601, 952

Shirley, Y. L., Evans, N. J., II, Young, K. E., Knez, C., \& Jaffe, D. T. 2003, ApJS, 149, 375

Shu, F., Najita, J., Ostriker, E., Wilkin, F., Ruden, S., \& Lizano, S. 1994, ApJ, 429, 781

Sollins, P. K., Zhang, Q., Keto, E., \& Ho, P. T. P. 2005, ApJ, 624, L49

Tielens, A. G. G. M. 2005, The Physics and Chemistry of the Interstellar Medium (Cambridge University Press) ApJ, 537, 283

van Dishoeck, E. F., \& Blake, G. A. 1998, ARA\&A, 36, 317

Viti, S., \& Williams, D. A. 1999, MNRAS, 310, 517

Viti, S., \& Williams, D. A. 1999, MNRAS, 305, 755

Vogel, S. N., Wright, M. C. H., Plambeck, R. L., \& Welch, W. J. 1984, ApJ, 283, 655

Yorke, H. W. 2002, ASP Conf. Ser. 267: Hot Star Workshop III: The Earliest Phases of Massive Star Birth, 267, 165

Wang, Y., Jaffe, D. T., Evans, N. J., II, Hayashi, M., Tatematsu, K., \& Zhou, S. 1993, ApJ, 419,707

Wilson, T. L., \& Rood, R. 1994, ARA\&A, 32, 191

Wolfire, M. G., \& Koenigl, A. 1993, ApJ, 415, 204

Wood, D. O. S., \& Churchwell, E. 1989, ApJS, 69, 831

Wu, Y., Wei, Y., Zhao, M., Shi, Y., Yu, W., Qin, S., \& Huang, M. 2004, A\&A, 426, 503 
Table 1. Observed Sample of Massive Star Forming Regions.

\begin{tabular}{|c|c|c|c|c|c|c|c|c|}
\hline \multirow[t]{2}{*}{ Name } & \multicolumn{2}{|c|}{ Position (J2000) } & \multicolumn{2}{|c|}{ RMS noise limit $(\mathrm{K})$} & \multirow{2}{*}{$\begin{array}{c}\mathrm{V}_{\mathrm{LSR}} \\
\left(\mathrm{km} \mathrm{s}^{-1}\right)\end{array}$} & \multicolumn{2}{|c|}{ Distance } & \multirow[t]{2}{*}{ P92 Name } \\
\hline & RA & DEC & $347 \mathrm{GHz}$ & $356 \mathrm{GHz}$ & & (kpc) & ref & \\
\hline G5.89 & 180030.3 & -240358 & 0.060 & 0.111 & 9 & 2 & 1 & W28A2 (1) \\
\hline G5.97 & 180340.4 & -242244 & 0.044 & 0.069 & 10 & 2.7 & 5 & $\ldots$ \\
\hline G8.67 & 180619.0 & -213732 & 0.068 & 0.074 & 36 & 8.5 & 2 & $8.67-0.36$ \\
\hline G10.47 & 180838.4 & -195152 & $\ldots \mathrm{b}$ & 0.100 & 67 & 12 & 2 & W31 (1) \\
\hline G12.21 & 181239.7 & -182421 & 0.042 & 0.076 & 24 & 16.3 & 2 & $12.21-0.1$ \\
\hline M17S & 182024.8 & -161135 & 0.044 & 0.077 & 20 & 2.3 & 5 & M17 (2) \\
\hline G19.61 & 182738.1 & -115640 & 0.048 & 0.065 & 43 & 4.5 & 1 & $19.61-0.23$ \\
\hline G20.08 & 182810.4 & -112849 & 0.044 & 0.068 & 42 & 4.1 & 1 & $20.08-0.13$ \\
\hline G29.96 & 184603.9 & -023922 & 0.044 & 0.079 & 98 & 9 & 1 & W43S \\
\hline G31.41 & 184733.0 & -011236 & 0.044 & 0.061 & 97 & 8.5 & 1 & $31.41+0.31$ \\
\hline G34.26 & 185318.5 & 011458 & 0.047 & 0.131 & 58 & 3.7 & 1 & W44 \\
\hline $\mathrm{G} 45.07^{\mathrm{c}}$ & 191322.1 & 105053 & 0.044 & 0.073 & 59 & 9.7 & 1 & $45.07+0.13$ \\
\hline G45.47 & 191425.6 & 110926 & 0.037 & 0.063 & 58 & 8.3 & 6 & $\ldots$ \\
\hline G61.48 & 194649.2 & 251248 & 0.037 & 0.048 & 12 & 2 & 1 & S88 B \\
\hline K3-50A & 200145.6 & 333242 & 0.035 & 0.077 & -24 & 8.6 & 3 & K3-50 \\
\hline G75.78 & 202144.1 & 372640 & 0.038 & 0.068 & 0 & 5.6 & 1 & $\mathrm{ON} 2 \mathrm{~N}$ \\
\hline Cep A & 225617.9 & 620149 & 0.031 & 0.076 & -10 & 0.7 & 1 & CEP A \\
\hline $\mathrm{W} 3(\mathrm{OH})$ & 022703.8 & 615225 & 0.026 & 0.079 & -48 & 2.4 & 2 & W3 (OH) \\
\hline G138.3 & 030129.2 & 602912 & 0.066 & 0.073 & -38 & 3.8 & 1 & S201 \\
\hline G139.9 & 030723.9 & 583053 & 0.058 & 0.071 & -39 & 4.2 & 1 & $\ldots$ \\
\hline G192.58 & 061253.6 & 175927 & 0.037 & 0.053 & 9 & 2.5 & 3 & $\mathrm{~S} 255 / 7$ \\
\hline G192.6 & 061253.6 & 180026 & 0.074 & 0.098 & 9 & 2.5 & 3 & $\mathrm{~S} 255 / 7$ \\
\hline G240.3 & 074451.9 & -240740 & 0.035 & 0.048 & 68 & 6.4 & 4 & $\ldots$ \\
\hline
\end{tabular}

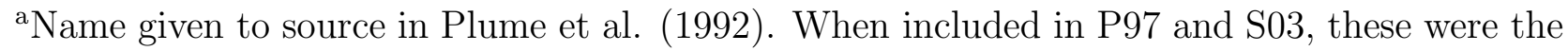
source names used.

${ }^{\mathrm{b}}$ Too much chemistry in spectrum to determine a reliable rms noise level. 
${ }^{\mathrm{c}}$ The rms noise limits for the two maps of this source are $0.1 \mathrm{~K}$ and $0.14 \mathrm{~K}$ for the $347 \mathrm{GHz}$ and $356 \mathrm{GHz}$ pointings respectively.

Note. - The source velocity here is the reference velocity of each source, which has been removed from each spectrum. RMS noise levels are given in $\mathrm{T}_{\mathrm{MB}}$. References:(1) Hanson et al. 2002, (2) S03, (3) Kurtz et al. 1994, (4) Kumar et al. 2003., (5) Churchwell et al. 1990, and (6) Hofner et al. 2000 
Table 2. Integrated Intensities for $\mathrm{SiO}, \mathrm{HCO}^{+}$and $\mathrm{H}^{13} \mathrm{CO}^{+}$.

\begin{tabular}{lccccccccc}
\hline \hline \multirow{2}{*}{ Name } & \multicolumn{3}{c}{$\mathrm{SiO}$ Properties } & \multicolumn{3}{c}{$\mathrm{HCO}^{+}$Properties } & \multicolumn{3}{c}{$\mathrm{H}^{13} \mathrm{CO}^{+}$Properties } \\
\cline { 2 - 9 } & $\mathrm{T}_{\mathrm{MB}}$ & $\int \mathrm{T}_{\mathrm{MB}} d v$ & $d v$ & $\mathrm{~T}_{\mathrm{MB}}$ & $\int \mathrm{T}_{\mathrm{MB}} d v$ & $d v$ & $\mathrm{~T}_{\mathrm{MB}}$ & $\int \mathrm{T}_{\mathrm{MB}} d v$ & $d v$ \\
\hline G5.89 & 2.3 & $75.3 \pm 0.7$ & 120 & 39.2 & $690.0 \pm 0.8$ & 105 & 8.9 & $65.2 \pm 0.4$ & 35 \\
G5.97 & $<0.1$ & $<0.3 \pm 0.1$ & 8 & 12.4 & $50.5 \pm 0.2$ & 15 & 0.6 & $1.6 \pm 0.1$ & 8 \\
G8.67 & 0.2 & $1.6 \pm 0.3$ & 13 & 13.8 & $80.0 \pm 0.3$ & 38 & 5.6 & $20.9 \pm 0.2$ & 10 \\
G10.47 & 1.0 & $9.9 \pm \cdots$ & 22 & 7.7 & $79.3 \pm 0.4$ & 35 & 1.2 & $9.2 \pm \cdots$ & 15 \\
G12.21 & 0.2 & $2.5 \pm 0.2$ & 25 & 10.0 & $93.8 \pm 0.3$ & 30 & 0.8 & $6.5 \pm 0.2$ & 15 \\
M17S & $<0.1$ & $<0.7 \pm 0.2$ & 15 & 16.2 & $79.0 \pm 0.2$ & 15 & 2.9 & $9.4 \pm 0.2$ & 11 \\
G19.61 & 0.8 & $15.9 \pm 0.3$ & 45 & 10.8 & $162.2 \pm 0.4$ & 55 & 1.8 & $16.1 \pm 0.2$ & 20 \\
G20.08 & 0.4 & $6.9 \pm 0.3$ & 40 & 6.5 & $75.0 \pm 0.3$ & 40 & 1.1 & $9.7 \pm 0.2$ & 25 \\
G29.96 & 0.5 & $7.9 \pm 0.3$ & 40 & 23.6 & $160.4 \pm 0.3$ & 30 & 3.8 & $13.2 \pm 0.1$ & 10 \\
G31.41 & $<0.1$ & $<0.7 \pm 0.2$ & 15 & 1.6 & $6.8 \pm 0.2$ & 13 & $<0.1$ & $<0.3 \pm 0.1$ & 3 \\
G34.26 & 1.3 & $26.0 \pm 0.4$ & 55 & 27.8 & $228.2 \pm 0.6$ & 35 & 4.3 & $33.2 \pm 0.2$ & 25 \\
G45.07 & 0.7 & $10.3 \pm 0.3$ & 37 & 15.0 & $169.4 \pm 0.4$ & 45 & 1.5 & $11.2 \pm 0.3$ & 33 \\
G45.47 & $<0.1$ & $<0.3 \pm 0.1$ & 12 & 10.1 & $59.4 \pm 0.2$ & 20 & 1.3 & $6.1 \pm 0.1$ & 12 \\
G61.48 & $<0.1$ & $<0.3 \pm 0.1$ & 8 & 10.8 & $58.5 \pm 0.1$ & 18 & 1.5 & $4.7 \pm 0.1$ & 8 \\
K3-50A & 0.3 & $2.1 \pm 0.1$ & 16 & 18.6 & $154.8 \pm 0.3$ & 25 & 1.8 & $10.6 \pm 0.2$ & 18 \\
G75.78 & 0.3 & $2.2 \pm 0.1$ & 13 & 12.5 & $136.8 \pm 0.3$ & 45 & 2.5 & $10.3 \pm 0.2$ & 17 \\
Cep A & 0.5 & $7.3 \pm 0.2$ & 45 & 23.2 & $234.7 \pm 0.4$ & 60 & 4.5 & $23.4 \pm 0.2$ & 28 \\
W3(OH) & 1.4 & $14.5 \pm 0.2$ & 50 & 18.5 & $148.2 \pm 0.3$ & 30 & 2.4 & $11.6 \pm 0.1$ & 15 \\
G138.3 & $<0.2$ & $<0.3 \pm 0.1$ & 2 & 5.0 & $10.6 \pm 0.2$ & 8 & 0.3 & $0.5 \pm 0.1$ & 4 \\
G139.9 & $<0.2$ & $<0.3 \pm 0.1$ & 11 & 8.6 & $18.8 \pm 0.1$ & 6 & $<0.2$ & $<0.3 \pm 0.1$ & 2 \\
G192.58 & 0.3 & $1.3 \pm 0.1$ & 8 & 14.5 & $84.2 \pm 0.2$ & 25 & 1.1 & $5.5 \pm 0.1$ & 15 \\
G192.6 & $<0.2$ & $<0.6 \pm 0.2$ & 30 & 12.0 & $54.1 \pm 0.3$ & 15 & 0.8 & $2.2 \pm 0.2$ & 8 \\
G240.3 & $<0.1$ & $<0.4 \pm 0.2$ & 10 & 7.3 & $59.1 \pm 0.2$ & 30 & 0.4 & $2.5 \pm 0.1$ & 14 \\
\hline
\end{tabular}

Note. - For the sources in which we did not detect $\mathrm{SiO}$ or $\mathrm{H}^{13} \mathrm{CO}^{+}, 3 \sigma$ upper limits on the brightness temperature and integrated intensities are given. 
Table 3. Observed and Derived Source Parameters.

\begin{tabular}{|c|c|c|c|c|c|c|c|c|}
\hline \multirow[t]{2}{*}{ Name } & \multicolumn{2}{|c|}{ Detection } & \multirow[t]{2}{*}{$f^{\mathrm{a}}$} & \multicolumn{2}{|c|}{ Column density $\left(\times 10^{12}\right)$} & \multirow[t]{2}{*}[\mathrm{SiO}]{$/\left[\mathrm{H}_{2}\right]^{\mathrm{b}}$} & \multirow{2}{*}{$\begin{array}{c}\mathrm{L}_{F I R} \\
\log \left(\mathrm{L}_{\odot}\right)\end{array}$} & \multirow{2}{*}{$\begin{array}{l}t_{\text {outflow }}{ }^{\mathrm{c}} \\
\left(10^{4} \mathrm{yr}\right)\end{array}$} \\
\hline & $\mathrm{SO}_{2}$ & Infall & & $\mathrm{H}^{13} \mathrm{CO}^{+}$ & $\mathrm{SiO}$ & & & \\
\hline G5.89 & $\mathrm{Y}$ & $\mathrm{N}$ & 0.79 & 39.7 & 421.0 & -8.90 & $5.25^{\mathrm{d}}$ & 0.2 \\
\hline G5.97 & $\mathrm{N}$ & $\mathrm{N}$ & 0.28 & 1.0 & $<1.7$ & $\cdots$ & $5.23^{\mathrm{d}}$ & $\cdots$ \\
\hline G8.67 & Y & Y & 0.24 & 12.7 & 9.0 & $\cdots$ & $5.70^{\mathrm{d}}$ & $\ldots$ \\
\hline G10.47 & Y & Y & 0.16 & 5.6 & 55.4 & -9.53 & $6.26^{\mathrm{d}}$ & $\ldots$ \\
\hline G12.21 & Y & $\mathrm{N}$ & 0.22 & 4.0 & 14.0 & -10.15 & $6.17^{\mathrm{d}}$ & $\ldots$ \\
\hline M17S & $\mathrm{N}$ & Y & 0.33 & 5.7 & $<3.9$ & $<-10.73$ & $5.72^{\mathrm{d}}$ & $\ldots$ \\
\hline G19.61 & Y & $\mathrm{Y}$ & 0.22 & 9.8 & 89.0 & -9.72 & $5.42^{\mathrm{d}}$ & $\ldots$ \\
\hline G20.08 & $\mathrm{Y}$ & $\mathrm{N}$ & 0.13 & 5.9 & 38.6 & -10.48 & $4.86^{\mathrm{d}}$ & $\ldots$ \\
\hline G29.96 & Y & $\mathrm{N}$ & 0.49 & 8.0 & 44.2 & -9.12 & $6.30^{\mathrm{d}}$ & $\ldots$ \\
\hline G31.41 & $\mathrm{N}$ & $\mathrm{N}$ & $\ldots$ & $<0.2$ & $<3.9$ & $<-11.89$ & $5.45^{\mathrm{d}}$ & $\ldots$ \\
\hline G34.26 & Y & Y & 0.58 & 20.2 & 145.0 & -10.43 & $5.77^{\mathrm{d}}$ & $\ldots$ \\
\hline G45.07 & $\mathrm{Y}$ & $\mathrm{N}$ & 0.32 & 6.8 & 57.6 & -9.54 & $6.15^{\mathrm{d}}$ & 4 \\
\hline G45.47 & Y & $\mathrm{N}$ & 0.21 & 3.7 & $<1.7$ & $\ldots$ & $6.04^{\mathrm{d}}$ & $\ldots$ \\
\hline G61.48 & $\mathrm{N}$ & $\mathrm{N}$ & 0.23 & 2.9 & $<1.7$ & $<-10.80$ & $5.01^{\mathrm{d}}$ & 7 \\
\hline K3-50A & Y & $\mathrm{N}$ & 0.40 & 6.5 & 11.7 & -11.60 & $6.35^{\mathrm{e}}$ & $\ldots$ \\
\hline G75.78 & Y & $\mathrm{N}$ & 0.25 & 6.3 & 12.3 & $\ldots$ & $5.65^{\mathrm{d}}$ & 3.7 \\
\hline Cep A & Y & Y & 0.47 & 14.2 & 40.8 & -9.78 & $4.40^{\mathrm{f}}$ & 0.2 \\
\hline $\mathrm{W} 3(\mathrm{OH})$ & $\mathrm{Y}$ & Y & 0.39 & 7.1 & 81.1 & -11.16 & $5.12^{\mathrm{e}}$ & $\ldots$ \\
\hline G138.3 & $\mathrm{N}$ & $\mathrm{N}$ & 0.11 & 0.3 & $<1.7$ & $\ldots$ & $4.57^{\mathrm{e}}$ & 17 \\
\hline G139.9 & $\mathrm{N}$ & $\mathrm{N}$ & $\ldots$ & $<0.2$ & $<1.7$ & $\ldots$ & $4.82^{\mathrm{e}}$ & 6 \\
\hline G192.58 & Y & Y & 0.32 & 3.4 & 7.3 & -10.94 & $4.79^{\mathrm{e}}$ & 50 \\
\hline G192.6 & Y & Y & 0.26 & 1.3 & $<3.4$ & $\ldots$ & $\ldots$ & $\ldots$ \\
\hline G240.3 & Y & Y & 0.16 & 1.5 & $<2.2$ & $\ldots$ & $\ldots$ & 2.3 \\
\hline
\end{tabular}

${ }^{a} \mathrm{HCO}^{+}$beam filling factor.

${ }^{\mathrm{b}} \mathrm{SiO}$ abundance relative to $\mathrm{H}_{2}$ for sources with CS observations (P97) and abundance calculations (S03).

${ }^{\mathrm{c}}$ Kinematic age of outflow from $\mathrm{Wu}$ et al. (2004). 
${ }^{\mathrm{d}}$ Far Infrared Luminosities modified from Wood \& Churchwell (1989).

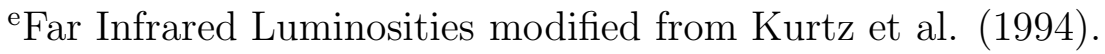

${ }^{\mathrm{f}}$ Far Infrared Luminosities taken from Evans et al. (1981). 
Table 4. Infall Velocities and Mass Infall Rates.

\begin{tabular}{lcccc}
\hline \hline Name & $\begin{array}{c}\mathrm{V}_{\text {in }}{ }^{\mathrm{a}} \\
\left(\mathrm{km} \mathrm{s}^{-1}\right)\end{array}$ & $\begin{array}{c}n_{\mathrm{H}_{2}}{ }^{\mathrm{b}} \\
\left(10^{5} \mathrm{~cm}^{-1}\right)\end{array}$ & $\left.\begin{array}{c}\dot{\mathrm{M}}_{\text {in }}{ }^{\mathrm{c}} \\
\left(10^{-4}\right.\end{array} \mathrm{M}_{\odot} \mathrm{yr}^{-1}\right)$ \\
\hline G8.67 & $0.4 \pm 0.1$ & 1.8 & $4 \pm$ & 2 \\
G10.47 & $1.8 \pm 0.3$ & 7.2 & $100 \pm$ & 80 \\
M17S & $1.4 \pm 0.5$ & 5.0 & $4 \pm$ & 2 \\
Cep A & $0.23 \pm 0.07$ & 10.0 & $0.17 \pm$ & 0.07 \\
W3(OH $)$ & $0.06 \pm 0.02$ & 60.0 & $3 \pm$ & 1 \\
G192.58 & $0.8 \pm 0.3$ & $\ldots$ & \multicolumn{3}{c}{$\ldots$} & \\
G192.6 & $0.9 \pm 0.4$ & 4.0 & $2 \pm$ & 1 \\
G34.26 & $1.5 \pm 0.3$ & 3.6 & $14 \pm$ & 4 \\
\hline
\end{tabular}

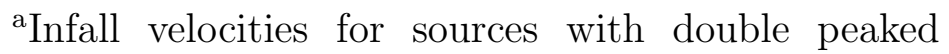
$\mathrm{HCO}^{+}$profiles.

${ }^{\mathrm{b}}$ Ambient densities taken from P97 except for: G34.26 (Hofner et al. 2000), M17S (Wang et al. 1993).

${ }^{\mathrm{c}}$ Mass infall rates. 


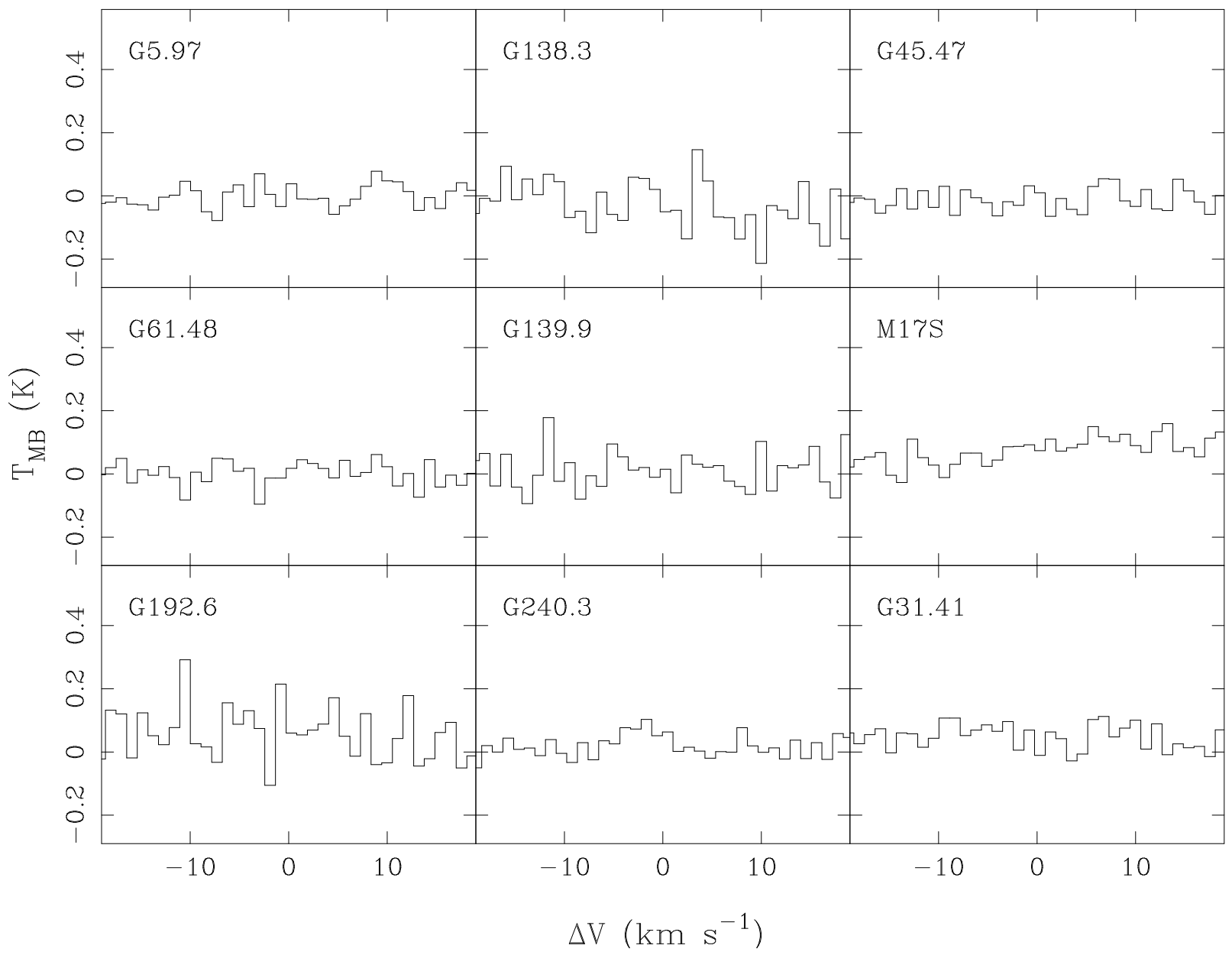

Fig. 1. - Nine sources in which $\mathrm{SiO}$ was not detected (to $4 \sigma$ limits). The name of each source is given in the top left hand corner of each panel. For each source, the source rest velocity is plotted as $\Delta v=0 \mathrm{~km} \mathrm{~s}^{-1}$. 


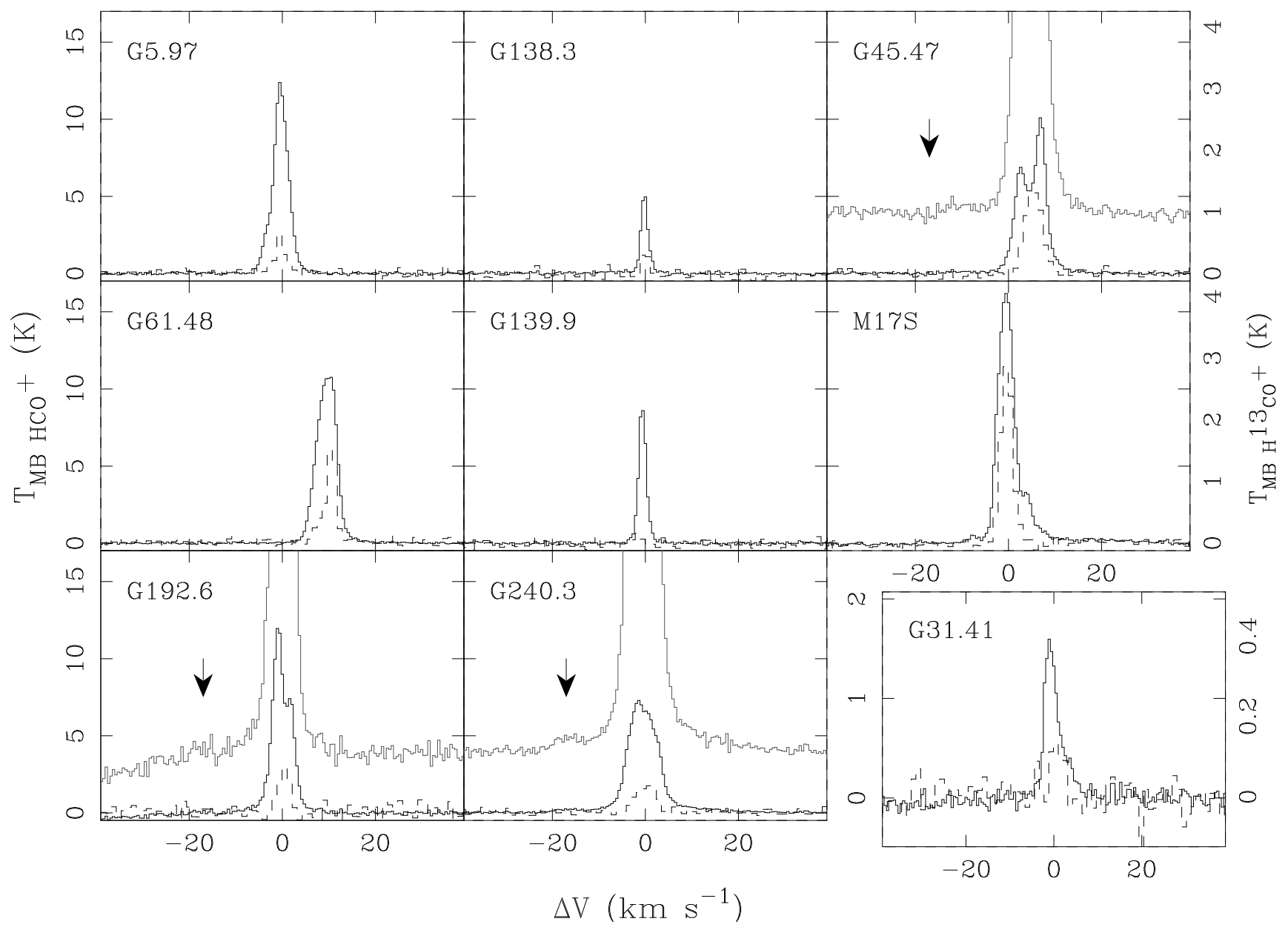

Fig. 2.- $\mathrm{HCO}^{+}$and $\mathrm{H}^{13} \mathrm{CO}^{+}$sources with no $\mathrm{SiO}$ detections (the same sources as in Figure 1). Solid lines show $\mathrm{HCO}^{+}$emission, while dashed lines show $\mathrm{H}^{13} \mathrm{CO}^{+}$emission scaled up by a factor of four. The temperature scale on the left hand side of the panels is the scale used for the $\mathrm{HCO}^{+}$spectra, and the temperature scale on the right hand side is that used for the $\mathrm{H}^{13} \mathrm{CO}^{+}$spectra. For sources with $\mathrm{SO}_{2}$ detections, we have re-plotted the $\mathrm{HCO}^{+}$spectra on a larger intensity scale ( from $-1 \mathrm{~K}$ to $3 \mathrm{~K}$, in gray) to show the low lying $\mathrm{SO}_{2}$ emission. The $\mathrm{SO}_{2}$ emission, when present, is centered at $-17 \mathrm{~km} \mathrm{~s}^{-1}$, and is indicated by an arrow. G31.41 is slightly offset to emphasize that the temperature scale has been magnified to show the emission feature. 


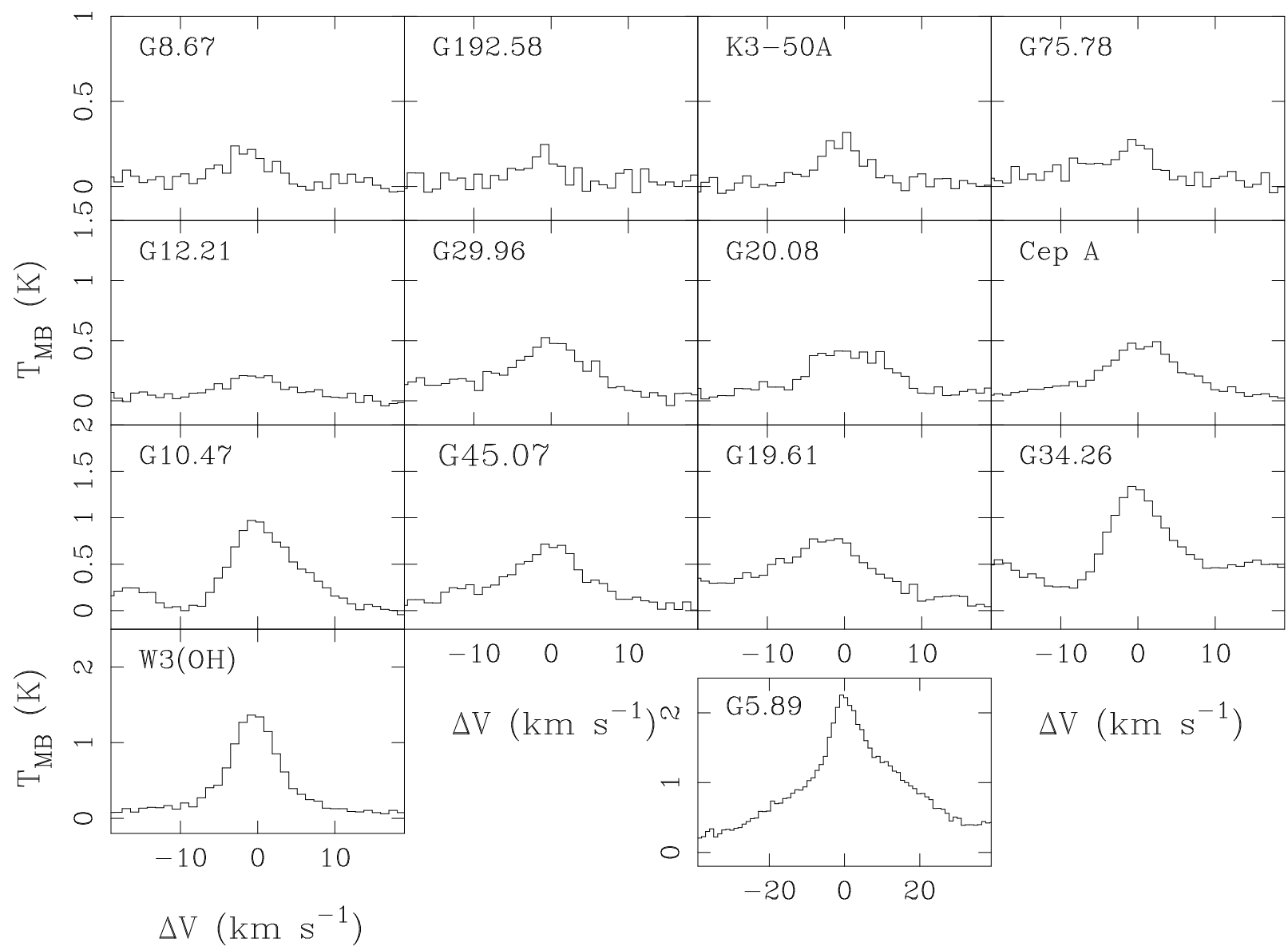

Fig. 3.- $\mathrm{SiO}$ spectra for 14 source in which $\mathrm{SiO}$ was detected (above $4 \sigma$ limits). For this figure only, the range of temperatures plotted increases by row. The plotted temperature ranges for each row increase by $0.5 \mathrm{~K}$ per row. The temperature scale in the top row extends from 0.2 to $1 \mathrm{~K}$ (in the $T_{\mathrm{MB}}$ scale), while the temperature scale in the bottom row extends from 0.2 to $2.5 \mathrm{~K}$. Note that the velocity scale for G5.89 is much wider (a $78 \mathrm{~km} \mathrm{~s}^{-1}$ window as opposed to a $38 \mathrm{~km} \mathrm{~s}^{-1}$ window for the other sources) in order to show the full width of the emission line, but that the temperature scale is the same as that for $\mathrm{W} 3(\mathrm{OH})$. 


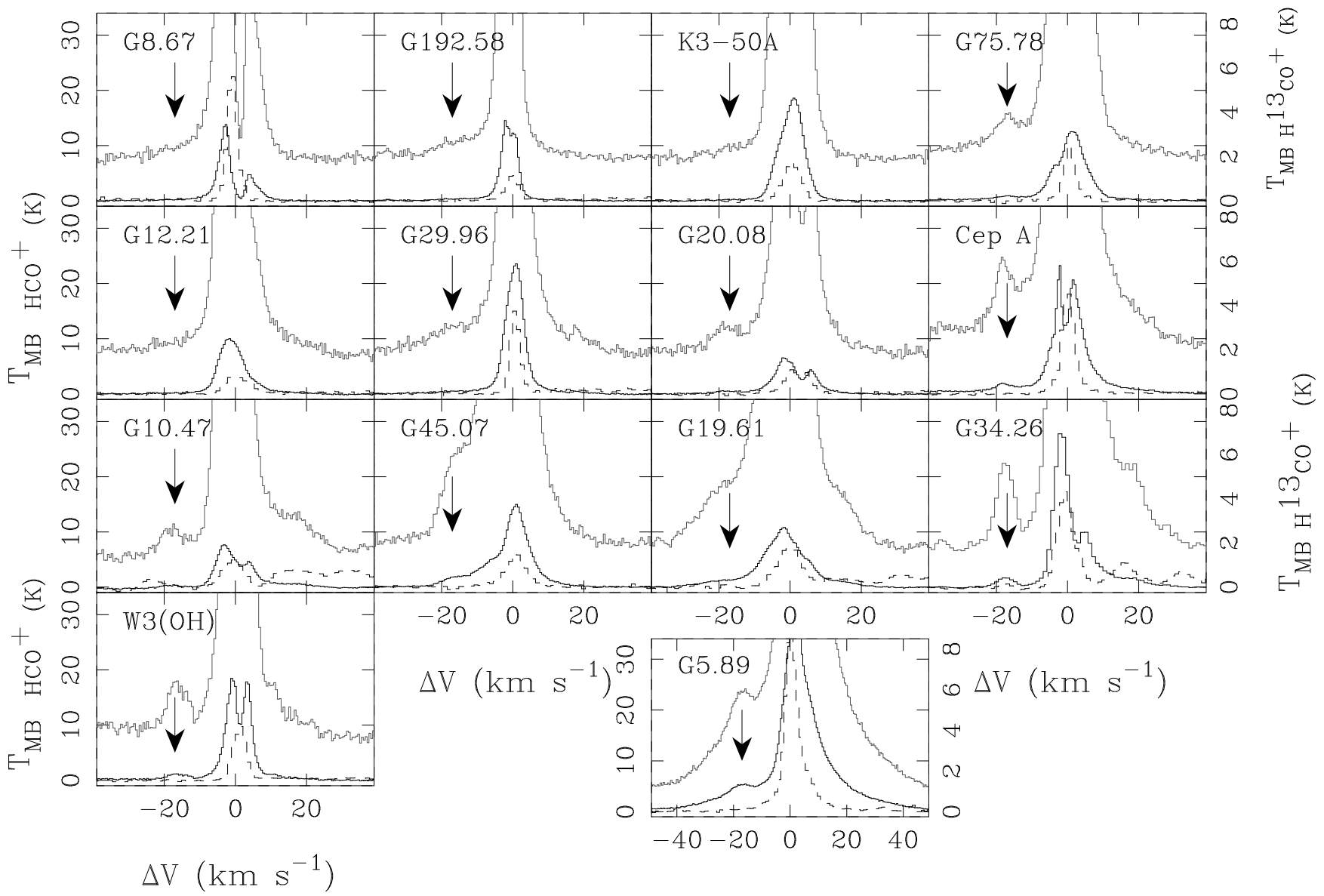

Fig. 4.- $\mathrm{HCO}^{+}$and $\mathrm{H}^{13} \mathrm{CO}^{+}$emission from sources with $\mathrm{SiO}$ detections (the same sources as in Figure 3). The solid, dashed and gray lines are the same as shown in Figure 2, as is the placement of the temperature scales. As in Figure 3, G5.89 has been plotted separately from the rest of the sources to stress that the velocity scale is larger for this source. 


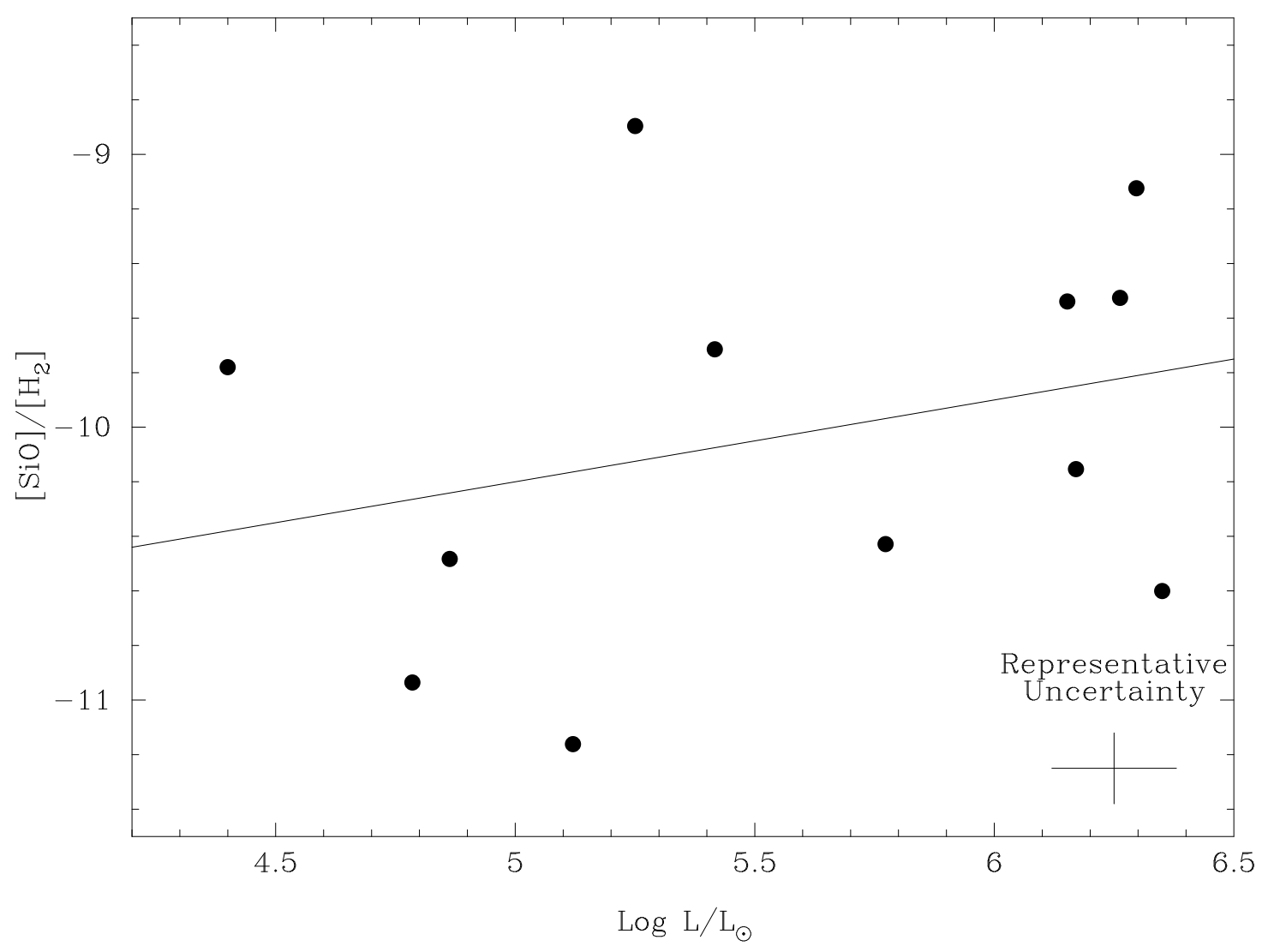

Fig. 5. - $\mathrm{SiO}$ abundance plotted as a function of source luminosity. The line of best fit shown is given by $\left(\log \left([\mathrm{SiO}] /\left[\mathrm{H}_{2}\right]\right)=0.30 \pm 0.06 \mathrm{Log}\left(L / L_{\odot}\right)-11.7 \pm 0.3\right)$, and was calculated only for sources detected in $\mathrm{SiO}$. This shows that $\mathrm{SiO}$ abundance increases with source luminosity. This is contrary to what is expected for $\mathrm{SiO}$ produced in PDRs, suggesting the observed $\mathrm{SiO}$ is produced in outflows. 


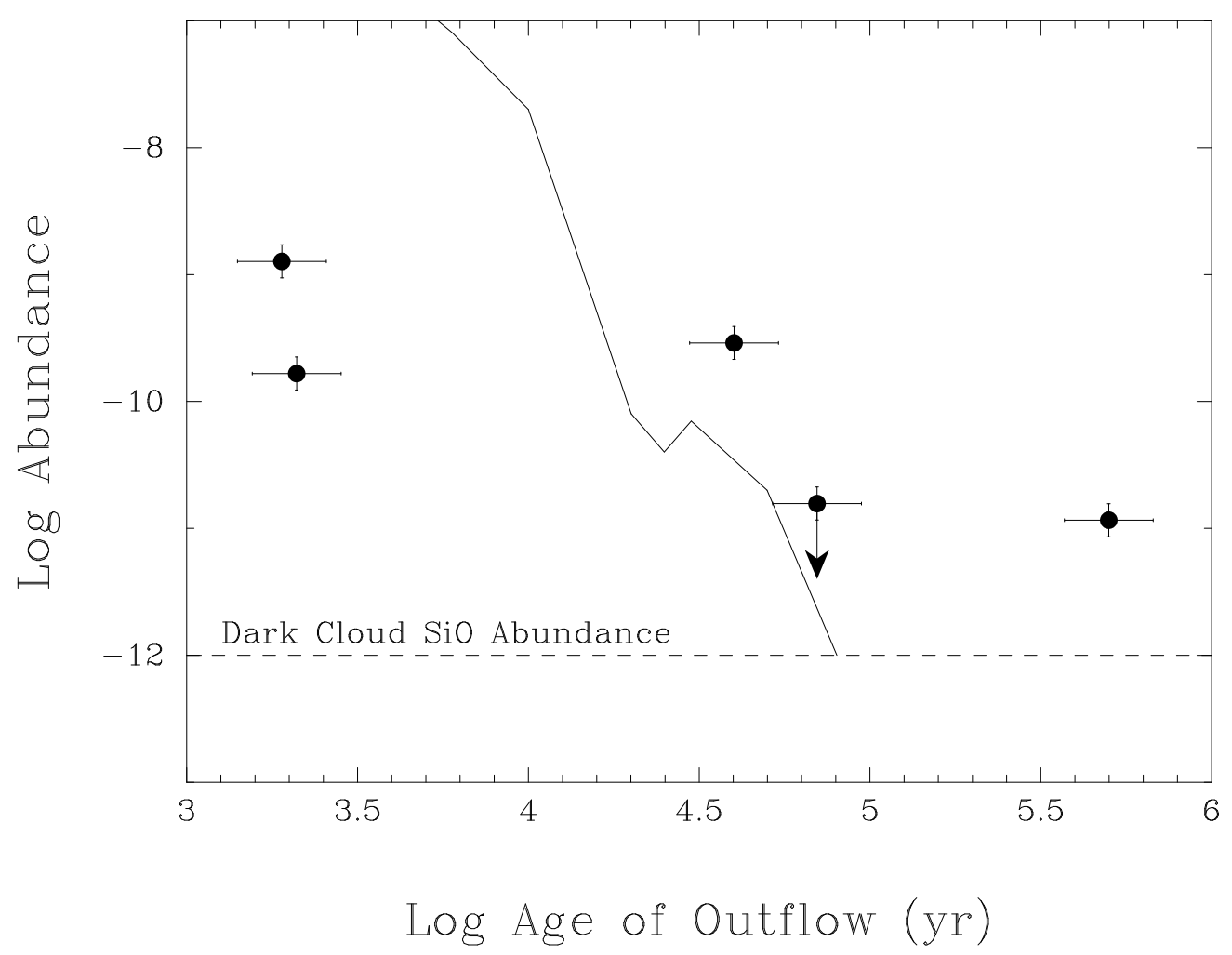

Fig. 6. - SiO abundance plotted as a function of outflow age. The outflow ages were taken from Wu et al. (2004). The solid line shows the model predictions of Pineau des Foréts et al. (1997) for the $\mathrm{SiO}$ abundance as a function of age (assuming Si returns to the dust grains), and the dashed line represents the canonical dark cloud abundance of SiO. The source shown with a downwards arrow represents G61.48, a source in which we did not detect $\mathrm{SiO}$, and the value given is an upper limit to the $\mathrm{SiO}$ abundance. The error bars represent $30 \%$ calibration uncertainty between our observations and those of P97 and S03 

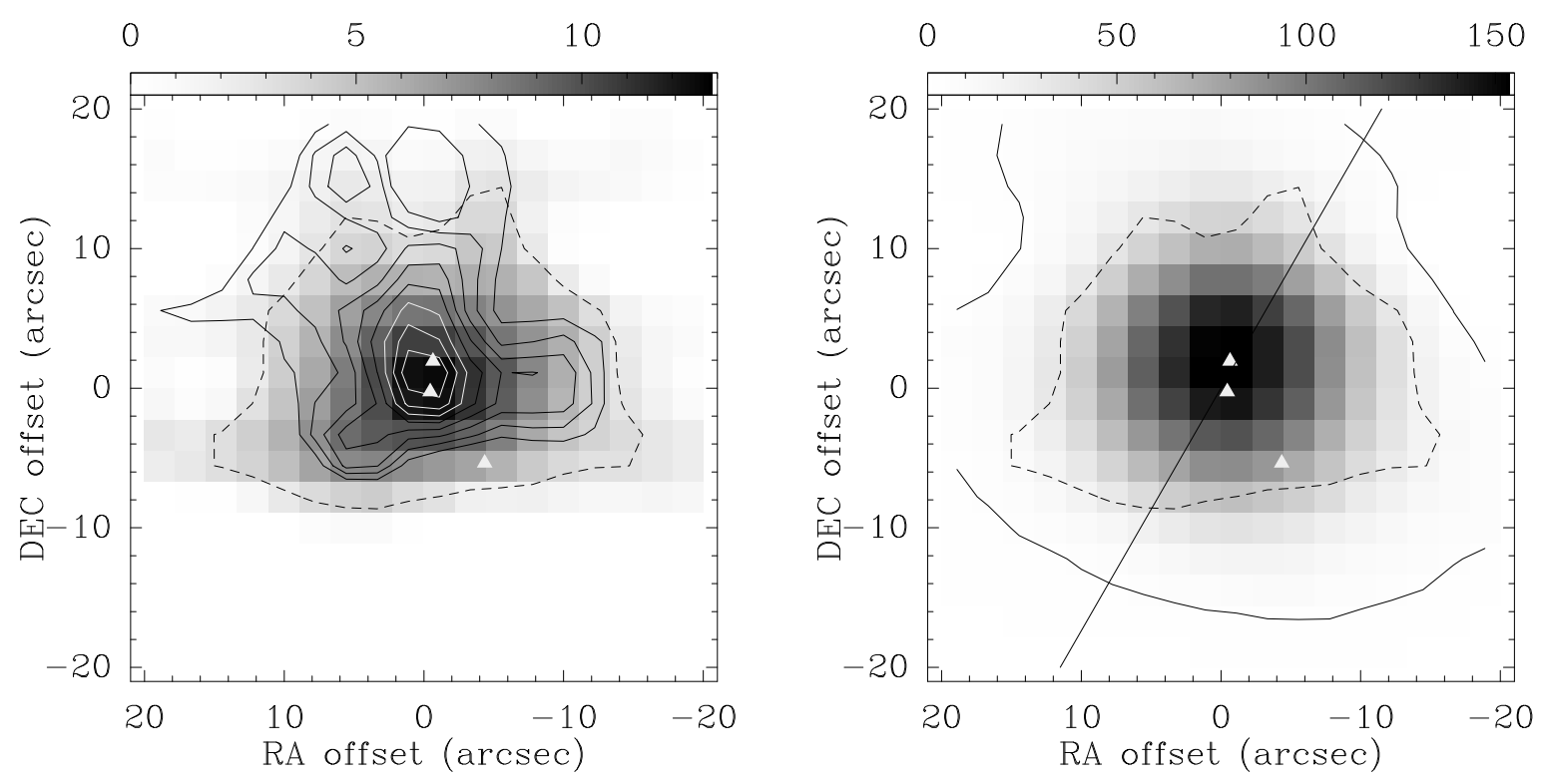

Fig. 7.- Left: $\mathrm{SiO}$ and $\mathrm{H}^{13} \mathrm{CO}^{+}$towards G45.07. The halftone scale represents the integrated $\mathrm{H}^{13} \mathrm{CO}^{+}$emission, while the contours show the integrated intensity of $\mathrm{SiO}$. The velocity range used to determine the integrated intensities is the same as the single pointing velocity range $(d v)$, however the rms noise limits are those listed at the end of Section 2. The first $\mathrm{SiO}$ contour is $5 \sigma\left(3.2 \mathrm{~K} \mathrm{~km} \mathrm{~s}^{-1}\right)$, incrementing in steps of $2 \sigma$, with the same scale continuing into the white contours near the center. Right: $\mathrm{HCO}^{+}$integrated intensity towards G45.07. The solid contour represents the $5 \sigma$ emission for the $\mathrm{HCO}^{+}$emission $(3.5$ $\mathrm{K} \mathrm{km} \mathrm{s}^{-1}$ ), and the solid line represents the cut used for the PV diagram along the outflow axis $\left(\mathrm{PA}=-30^{\circ}\right)$ presented in Figure 8, For both panels, the dashed line represents the $5 \sigma$ $\mathrm{H}^{13} \mathrm{CO}^{+}$emission contour $\left(3.0 \mathrm{~K} \mathrm{~km} \mathrm{~s}^{-1}\right)$. The three triangles represent the Mid IR sources detected by De Buizer et al. (2005), with the two points in the center corresponding to 230 $\mathrm{GHz}$ continuum sources detected at the Submillimeter Array (Klaassen et al. in prep). 

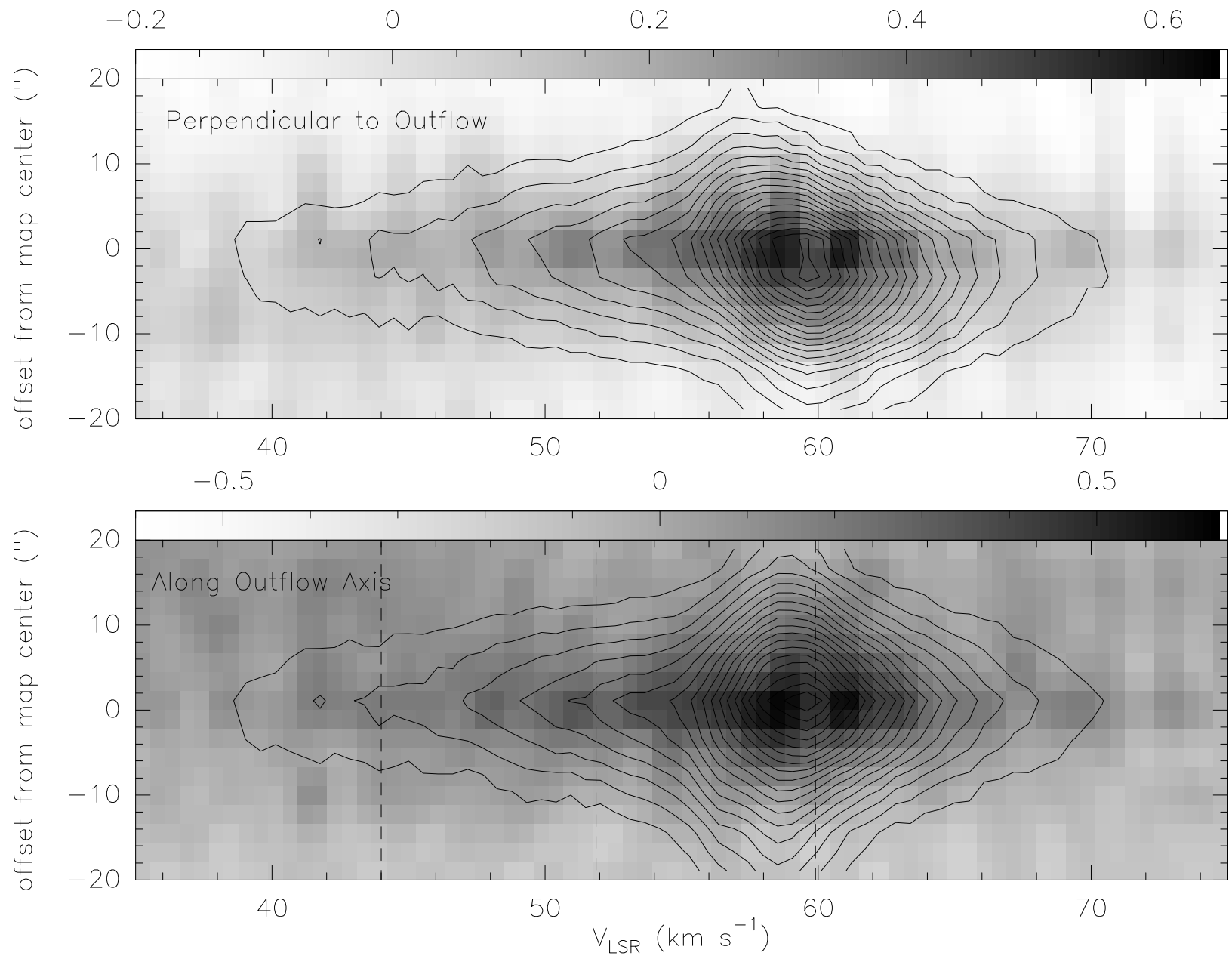

Fig. 8.- Position-Velocity (PV) diagrams for $\mathrm{SiO}$ (halftone scale) and $\mathrm{HCO}^{+}$emission (contours) in G45.07 both perpendicular to (top panel) and along the outflow axis (bottom panel) as defined in Hunter et al. (1997). These PV diagrams were taken at positions angles of $60^{\circ}$ and $-30^{\circ}$ east of north respectively, with the cut for the bottom panel of this figure shown in the right panel of Figure 7 . The first contour for $\mathrm{HCO}^{+}$is $5 \sigma$, or $0.7 \mathrm{~K}$ since the rms noise limit for this map is $0.14 \mathrm{~K}$, and the contours increase in increments of $5 \sigma$. The three dashed lines in the bottom panel show the peak velocities of the Gaussian fits to the $\mathrm{HCO}^{+}$spectra. 


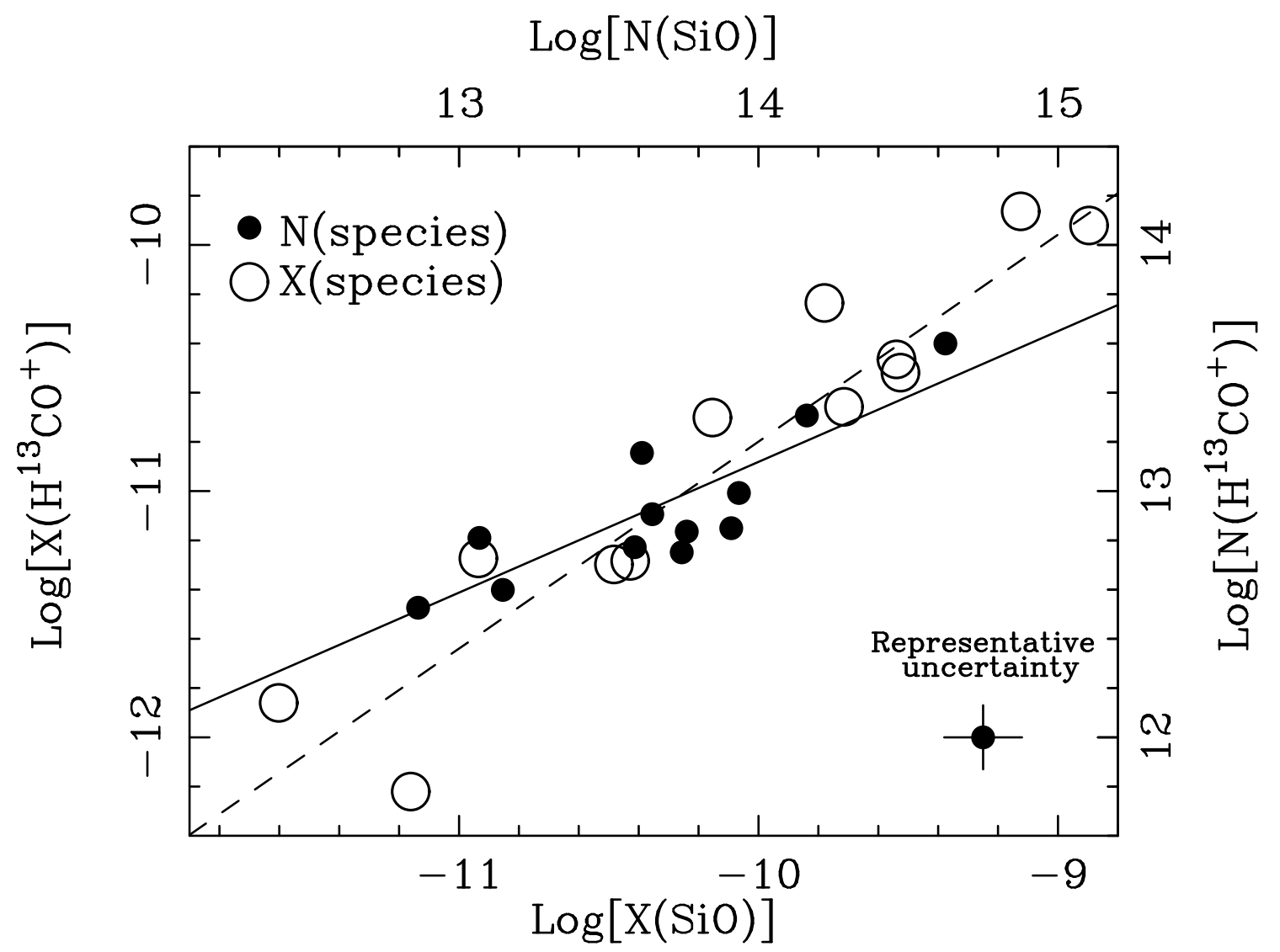

Fig. 9.- The abundance of $\mathrm{H}^{13} \mathrm{CO}^{+}$with respect to the abundance of $\mathrm{SiO}$ (open circles, dashed line of best fit) appears to increase faster than the respective column densities of these two species (filled circles, solid line of best fit). The equations of the two lines of best fit are: $\log \left[\mathrm{X}\left(\mathrm{H}^{13} \mathrm{CO}^{+}\right)\right]=(0.84 \pm 0.09) \log [\mathrm{X}(\mathrm{SiO})]-(2.4 \pm 0.9)$, and $\log \left[\mathrm{N}\left(\mathrm{H}^{13} \mathrm{CO}^{+}\right)\right]=(0.5 \pm 0.1) \log [\mathrm{N}(\mathrm{SiO})]+(5 \pm 1)$. This relationship could be due to $\mathrm{HCO}^{+}$ being enhanced (similarly to $\mathrm{SiO}$ ), as discussed in the text. 